\title{
The Study of the Solution to a Generalized KdV-mKdV Equation
}

\author{
Xiumei Lv, ${ }^{1}$ Tengwei Shao, ${ }^{1}$ and Jiacheng Chen ${ }^{2}$ \\ ${ }^{1}$ School of Finance, Chongqing Technology and Business University, Chongqing 400067, China \\ ${ }^{2}$ School of Finance, Southwestern University of Finance and Economics, Chengdu 611130, China \\ Correspondence should be addressed to Xiumei Lv; lvxium@126.com
}

Received 26 April 2013; Accepted 2 June 2013

Academic Editor: Shaoyong Lai

Copyright (C) 2013 Xiumei Lv et al. This is an open access article distributed under the Creative Commons Attribution License, which permits unrestricted use, distribution, and reproduction in any medium, provided the original work is properly cited.

\begin{abstract}
A mathematical technique based on an auxiliary equation and the symbolic computation system Matlab is employed to investigate a generalized KdV-mKdV equation which possesses high-order nonlinear terms. Some new solutions including the Jacobi elliptic function solutions, the degenerated soliton-like solutions, and the triangle function solutions to the equation are obtained.
\end{abstract}

\section{Introduction}

Many powerful techniques have been established during the past decades for the study of the nonlinear dispersive partial differential equations [1-9]. The inverse scattering method, the Backlund transformation, the Darboux transformation, the Painleve' analysis, the pseudospectral method, the finite differences method, and the sine-cosine ansatz are used to acquire solitary wave solutions and compactons solutions for some nonlinear equations. Wadati [1] employed the potential function to handle the $\mathrm{KdV}$ equation, and the resulting equation was solved iteratively by making use of a formal series for the potential function. Wadati [2] developed the trace method to handle the KP equation. The tanh method, developed by Malfliet and Hereman [3], is heavily used in the literature to deal with nonlinear partial differential equations. Fan and Zhang [4] introduced a useful extended tanh method that combined the standard tanh method with the Riccati equation. The extensive method was effectively used by many researchers to investigate exact solutions for a lot of nonlinear models (see $[10,11]$ ).

With the development of the symbolic computation system, the direct methods for constructing travelling wave solutions to differential equations become feasible. With the help of Mathematica, Sirendaoreji and Jiong [12, 13] used the auxiliary equation method to investigate $\mathrm{KdV}$ and $\mathrm{mKdV}$ equations, Boussinesq equations, sine-Gordon equations, and the nonlinear Klein-Gordon equations, respectively. The Jacobi elliptic function expansion method is confirmed as a powerful technique to solve some nonlinear differential equations (see [14]).

Zhang et al. [15] used a sub-ODE technique to investigate the exact solution of the following nonlinear dispersive $\mathrm{KdV}$ mKdV equation:

$$
u_{t}+\left(\alpha+\beta u^{p}+\lambda u^{2 p}\right) u_{x}+u_{x x x}=0
$$

where $\alpha, \beta, \lambda$, and $p$ are constants. The bell type solitary wave solution, the kink type solitary wave solution, the algebraic solitary wave solution, and the sinusoidal travelling wave solution of (1) with exponent $p>0$ were expressed explicitly in [15]. In fact, (1) is turned into a $\mathrm{KdV}$ equation if $p=$ $1, \beta \neq 0, \lambda=0$, and an $\mathrm{mKdV}$ equation if $p=1, \beta=0, \lambda \neq 0$.

In this paper, we further develop the work in [15] for the study of (1). By using a mathematical technique different from those in previous works [1-10], we obtain the exact travelling wave solutions including Jacobi elliptic function solutions, degenerated soliton solutions, and triangle function solutions for (1) with exponent $p$. Many of the solutions obtained are different from those presented in Zhang et al.'s work [15].

\section{Brief Description of the Approach}

To illustrate the basic concept of the auxiliary differential method, we consider that the nonlinear partial differential equation has the form

$$
P\left(u, u_{t}, u_{x}, u_{x x}, u_{x t}, u_{t t}, \ldots\right)=0 .
$$


Using the transformation $\xi=\mu(x-c t)(\mu \neq 0$ and $c \neq 0)$, (2) turns into the following nonlinear ordinary different equation:

$$
Q\left(u, u_{\xi}, u_{\xi \xi}, u_{\xi \xi \xi}, \ldots\right)=0
$$

We seek for the solutions of (3) in the form

$$
u(\xi)=\sum_{i=0}^{N} g_{i} z^{i}(\xi)
$$

where $g_{i}(i=0,1,2, \ldots, N)$ are constants which will be determined later. The parameter $N$ is a positive integer and can be determined by balancing the highest-order derivative terms and the highest power nonlinear terms in (3). The highest degree of $\partial^{p} u / \partial \xi^{p}$ can be calculated by

$$
\begin{gathered}
O\left[\frac{\partial^{p} u}{\partial \xi^{p}}\right]=N+p, \quad p=0,1,2, \ldots, \\
O\left[u^{q} \frac{\partial^{p} u}{\partial \xi^{p}}\right]=q N+p, \quad q, p=0,1,2, \ldots
\end{gathered}
$$

We assume that $z(\xi)$ represents the solutions of the following auxiliary differential equation

$$
\left(\frac{d z}{d \xi}\right)^{2}=c_{1}+c_{2} z^{2}+\frac{c_{3}}{2} z^{4}
$$

where $c_{i}(i=1,2,3)$ are real constants.

Substituting (4) and (6) into (3) and equating the coefficients of all powers of $z(\xi)$ and $z^{j}(\xi) \sqrt{c_{1}+c_{2} z^{2}+\left(c_{3} / 2\right) z^{4}}(j=0,1,2, \ldots)$ to be zero in the resulting equation, several algebraic equations will be obtained. Then, solving these algebraic equations by the symbolic computation system Matlab and combining (4) and the solutions of the auxiliary equation (6), we can get the exact solutions for (2).

\section{Exact Travelling Wave Solutions to (1)}

Firstly, setting $u^{p}=w$ yields

$$
\begin{gathered}
u_{x}=\frac{1}{p} w^{1 / p-1} w_{x}, \quad u_{t}=\frac{1}{p} w^{1 / p-1} w_{t} \\
u_{x x}=\frac{1}{p}\left(\frac{1}{p}-1\right) w^{1 / p-2}\left(w_{x}\right)^{2}+\frac{1}{p} w^{1 / p-1} w_{x x}, \\
u_{x x x}=\frac{1}{p}\left(\frac{1}{p}-1\right)\left(\frac{1}{p}-2\right) w^{1 / p^{-3}} w_{x}^{3} \\
+\frac{3}{p}\left(\frac{1}{p}-1\right) w^{1 / p-2} w_{x} w_{x x} \\
+\frac{1}{p} w^{1 / p-1} w_{x x x},
\end{gathered}
$$

which turns (1) into

$$
\begin{gathered}
w^{2} w_{t}+\left(\alpha+\beta w+\lambda w^{2}\right) w^{2} w_{x}+\left(\frac{1}{p}-1\right)\left(\frac{1}{p}-2\right) w_{x}^{3} \\
+3\left(\frac{1}{p}-1\right) w w_{x} w_{x x}+w^{2} w_{x x x}=0 .
\end{gathered}
$$

To find the traveling solutions for (1), we use the wave variable $\xi=\mu(x-c t)$, where $c \neq 0$ and $\mu \neq 0$. The wave variable $\xi$ transforms (8) into the following ordinary differential equation:

$$
\begin{aligned}
\left(\alpha+\beta w+\lambda w^{2}\right) w^{2} w^{\prime}+\left(\frac{1}{p}-1\right)\left(\frac{1}{p}-2\right) \mu^{2} w^{\prime 3} \\
+3 \mu^{2}\left(\frac{1}{p}-1\right) w w^{\prime} w^{\prime \prime}-c w^{2} w^{\prime}+\mu^{2} w^{2} w^{\prime \prime \prime}=0 .
\end{aligned}
$$

From (9), we have $N=1$. Therefore, we choose the ansatz

$$
w(\xi)=g_{0}+g_{1} z
$$

where $z(\xi)$ may be determined by

$$
\left(\frac{d z}{d \xi}\right)^{2}=c_{1}+c_{2} z^{2}+\frac{c_{3}}{2} z^{4},
$$

which possesses several types of solutions listed in Table 1 (see [16]).

In Table 1, functions $\operatorname{sn}(\xi)=\operatorname{sn}(\xi, r), \operatorname{cn}(\xi)=\operatorname{cn}(\xi, r)$, and $\operatorname{dn}(\xi)=\operatorname{dn}(\xi, r)$, are Jacobian elliptic functions with modulus $r(0<r<1)$, which have the properties $\operatorname{sn}(-\xi)=$ $-\operatorname{sn}(\xi), \operatorname{cn}(-\xi)=\operatorname{cn}(\xi), \operatorname{dn}(-\xi)=\operatorname{dn}(\xi), \operatorname{sn}^{2}(\xi)+\operatorname{cn}^{2}(\xi)=$ $1, \mathrm{dn}^{2}(\xi)+r^{2} \operatorname{sn}^{2}(\xi)=1,(\operatorname{sn}(\xi))^{\prime}=\mathrm{cn}(\xi) \operatorname{dn}(\xi),(\mathrm{cn}(\xi))^{\prime}=$ $-\operatorname{sn}(\xi) \operatorname{dn}(\xi)$, and $(\operatorname{dn}(\xi))^{\prime}=-r^{2} \operatorname{sn}(\xi) \operatorname{cn}(\xi)$. Setting $r \rightarrow 0$ yields $\operatorname{sn}(\xi) \rightarrow \sin (\xi), \operatorname{cn}(\xi) \rightarrow \cos (\xi)$, and $\operatorname{dn}(\xi) \rightarrow 1$. When $r \rightarrow 1$, it derives that $\operatorname{sn}(\xi) \rightarrow \tanh (\xi), \operatorname{cn}(\xi) \rightarrow$ $\operatorname{sech}(\xi)$, and $\operatorname{dn}(\xi) \rightarrow \operatorname{sech}(\xi)$.

Substituting (10) and (11) into (9) and letting each coefficient of $z^{j} \sqrt{c_{1}+c_{2} z^{2}+\left(c_{3} / 2\right) z^{4}}(0 \leq j \leq 4)$ be zero, we obtain

$$
\begin{aligned}
\lambda g_{1}^{5}+ & \frac{\mu^{2}}{2}\left(\frac{1}{p}-1\right)\left(\frac{1}{p}-2\right) g_{1}^{3} c_{3} \\
& +3\left(\frac{1}{p}-1\right) \mu^{2} g_{1}^{3} c_{3}+3 \mu^{2} g_{1}^{3} c_{3}=0, \\
\left(\beta g_{1}\right. & \left.+2 \lambda g_{0} g_{1}\right) g_{1}^{3}+2 \lambda g_{0} g_{1}^{4} \\
& +3\left(\frac{1}{p}-1\right) \mu^{2} g_{0} g_{1}^{2} c_{3}+6 \mu^{2} g_{0} g_{1}^{2} c_{3}=0, \\
-c g_{1}^{3} & +\left(\alpha+\beta g_{0}+\lambda g_{0}^{2}\right) g_{1}^{3}+2\left(\beta g_{1}+2 \lambda g_{0} g_{1}\right) g_{0} g_{1}^{2} \\
& +\lambda g_{0}^{2} g_{1}^{3}+\mu^{2}\left(\frac{1}{p}-1\right)\left(\frac{1}{p}-2\right) g_{1}^{3} c_{2}
\end{aligned}
$$


TABLE 1

\begin{tabular}{|c|c|c|c|c|}
\hline No. & $z(\xi)$ & $c_{3}$ & $c_{2}$ & $c_{1}$ \\
\hline 1 & $\operatorname{sn}(\xi), \operatorname{cd}(\xi)=\frac{\operatorname{cn}(\xi)}{\operatorname{dn}(\xi)}$ & $2 r^{2}$ & $-\left(r^{2}+1\right)$ & 1 \\
\hline 2 & $\operatorname{cn}(\xi)$ & $-2 r^{2}$ & $2 r^{2}-1$ & $1-r^{2}$ \\
\hline 3 & $\operatorname{dn}(\xi)$ & -2 & $2-r^{2}$ & $r^{2}-1$ \\
\hline 4 & $\mathrm{nc}(\xi)=\frac{1}{\mathrm{cn}(\xi)}$ & $2\left(1-r^{2}\right)$ & $2 r^{2}-1$ & $-r^{2}$ \\
\hline 5 & $\mathrm{~ns}(\xi)=\frac{1}{\operatorname{sn}(\xi)}, \mathrm{dc}(\xi)=\frac{\operatorname{dn}(\xi)}{\operatorname{cn}(\xi)}$ & 2 & $-\left(r^{2}+1\right)$ & $r^{2}$ \\
\hline 6 & $\operatorname{nd}(\xi)=\frac{1}{\operatorname{dn}(\xi)}$ & $2\left(r^{2}-1\right)$ & $2-r^{2}$ & -1 \\
\hline 7 & $\operatorname{cs}(\xi)=\frac{\operatorname{cn}(\xi)}{\operatorname{sn}(\xi)}$ & 2 & $2-r^{2}$ & $1-r^{2}$ \\
\hline 8 & $\operatorname{sc}(\xi)=\frac{\operatorname{sn}(\xi)}{\operatorname{cn}(\xi)}$ & $2\left(1-r^{2}\right)$ & $2-r^{2}$ & 1 \\
\hline 9 & $\operatorname{sd}(\xi)=\frac{\operatorname{sn}(\xi)}{\operatorname{dn}(\xi)}$ & $2 r^{2}\left(r^{2}-1\right)$ & $2 r^{2}-1$ & 1 \\
\hline 10 & $\mathrm{ds}(\xi)=\frac{\operatorname{dn}(\xi)}{\operatorname{sn}(\xi)}$ & 2 & $2 r^{2}-1$ & $r^{4}-r^{2}$ \\
\hline 11 & $r \operatorname{cn}(\xi) \pm \operatorname{dn}(\xi)$ & $-\frac{1}{2}$ & $\frac{r^{2}+1}{2}$ & $-\frac{\left(1-r^{2}\right)^{2}}{4}$ \\
\hline 12 & $\frac{1}{\operatorname{sn}(\xi)} \pm \frac{\operatorname{cn}(\xi)}{\operatorname{sn}(\xi)}$ & $\frac{1}{2}$ & $\frac{-2 r^{2}+1}{2}$ & $\frac{1}{4}$ \\
\hline 13 & $\frac{1}{\operatorname{cn}(\xi)} \pm \frac{\operatorname{sn}(\xi)}{\operatorname{cn}(\xi)}$ & $\frac{1-r^{2}}{2}$ & $\frac{r^{2}+1}{2}$ & $\frac{1-r^{2}}{4}$ \\
\hline 14 & $\frac{1}{\operatorname{sn}(\xi)} \pm \frac{\operatorname{dn}(\xi)}{\operatorname{sn}(\xi)}$ & $\frac{1}{2}$ & $\frac{r^{2}-2}{2}$ & $\frac{r^{4}}{4}$ \\
\hline 15 & $\operatorname{sn}(\xi) \pm i \operatorname{cn}(\xi), \frac{\operatorname{dn}(\xi)}{[}$ & $\frac{r^{2}}{2}$ & $\underline{r^{2}-2}$ & $\frac{r^{2}}{1}$ \\
\hline 16 & $\begin{array}{c}\sqrt{1-r^{2}} \operatorname{sn}(\xi) \pm \operatorname{cn}(\xi) \\
r \operatorname{sn}(\xi) \pm i \operatorname{dn}(\xi), \frac{\operatorname{sn}(\xi)}{1 \pm \operatorname{cn}(\xi)}\end{array}$ & $\begin{array}{l}2 \\
\frac{1}{2}\end{array}$ & $\begin{array}{c}2 \\
\frac{1-2 r^{2}}{2}\end{array}$ & $\begin{array}{l}4 \\
\frac{1}{4}\end{array}$ \\
\hline 17 & $\frac{\operatorname{sn}(\xi)}{1 \pm \operatorname{dn}(\xi)}$ & $\frac{r^{2}}{2}$ & $\frac{r^{2}-2}{2}$ & $\frac{1}{4}$ \\
\hline 18 & $\operatorname{dn}(\xi)$ & $\underline{r^{2}-1}$ & $\underline{r^{2}+1}$ & $\underline{r^{2}-1}$ \\
\hline & $\begin{array}{l}1 \pm \operatorname{rsn}(\xi) \\
\operatorname{cn}(\xi)\end{array}$ & $\begin{array}{c}2 \\
1-r^{2}\end{array}$ & $r^{2}+1$ & $\begin{array}{c}4 \\
-r^{2}+1\end{array}$ \\
\hline 19 & $\overline{1 \pm \operatorname{sn}(\xi)}$ & $\overline{2}$ & $\overline{2}$ & 4 \\
\hline 20 & $\frac{\operatorname{sn}(\xi)}{\operatorname{dn}(\xi) \pm \operatorname{cn}(\xi)}$ & $\frac{\left(1-r^{2}\right)^{2}}{2}$ & $\frac{r^{2}+1}{2}$ & $\frac{1}{4}$ \\
\hline 21 & $\frac{\operatorname{cn}(\xi)}{\sqrt{1-r^{2}} \pm \operatorname{dn}(\xi)}$ & $\frac{r^{4}}{2}$ & $\frac{r^{2}-2}{2}$ & $\frac{1}{4}$ \\
\hline
\end{tabular}

$+3\left(\frac{1}{p}-1\right) \mu^{2} g_{1}^{3} c_{2}+3 c_{3} \mu^{2} g_{0}^{2}+\mu^{2} c_{2} g_{1}^{3}=0$,

$-2 c g_{0} g_{1}^{2}+2\left(\alpha+\beta g_{0}+\lambda g_{0}^{2}\right) g_{1}^{2} g_{0}+\left(\beta g_{1}+2 \lambda g_{0} g_{1}\right) g_{0}^{2} g_{1}$

$+3\left(\frac{1}{p}-1\right) \mu^{2} g_{1}^{2} g_{0} c_{2}+2 \mu^{2} g_{1}^{2} g_{0} c_{2}=0$,

$-c g_{0}^{2} g_{1}+\left(\alpha+\beta g_{0}+\lambda g_{0}^{2}\right) g_{0}^{2} g_{1}$

$+\left(\frac{1}{p}-1\right)\left(\frac{1}{p}-2\right) \mu^{2} g_{1}^{3} c_{1}+\mu^{2} g_{0}^{2} g_{1} c_{2}=0$.
Solving (12) with the help of Matlab, we acquire the following solutions:

$$
\begin{gathered}
p=1, \quad g_{0}=-\frac{\beta}{2 \lambda}, \quad g_{1}= \pm \frac{\beta}{2 \lambda} \sqrt{-\frac{c_{3}}{c_{2}}}, \\
\mu= \pm \frac{\beta}{2} \sqrt{\frac{1}{3 \lambda c_{2}}}, \quad c=\alpha-\frac{\beta^{2}}{6 \lambda}, \\
p=\frac{1}{2}, \quad g_{0}=-\frac{2 \beta}{5 \lambda}, \quad g_{1}= \pm \frac{2 \beta}{5 \lambda} \sqrt{-\frac{c_{3}}{c_{2}}},
\end{gathered}
$$




$$
\begin{gathered}
\mu= \pm \frac{\beta}{5} \sqrt{\frac{2}{3 \lambda c_{2}}}, \quad c=\alpha-\frac{16 \beta^{2}}{75 \lambda}, \\
p=2, \quad \mu= \pm\left(\frac{5 c_{2} \beta^{2}+5 \epsilon \beta^{2} \sqrt{c_{2}^{2}-2 c_{1} c_{3}}}{48 c_{1} c_{3} \lambda}\right)^{1 / 2}, \\
g_{0}=-\frac{5 \beta}{8 \lambda}, \quad g_{1}= \pm \frac{\mu}{4} \sqrt{-\frac{30 c_{3}}{\lambda}}, \quad c=\alpha-\frac{5 \beta^{2}}{32 \lambda}+\frac{1}{4} \mu^{2} c_{2}, \\
g_{0}=-\frac{\beta(2 p+1)}{2 \lambda(p+2)}, \quad g_{1}= \pm \frac{\beta(2 p+1)}{2 \lambda(p+2)} \sqrt{-\frac{c_{3}}{c_{2}}}, \quad 2 c_{1} c_{3}=c_{2}^{2}, \\
\mu= \pm \frac{\beta p}{p+2} \sqrt{\frac{2 p+1}{2 \lambda(p+1) c_{2}}}, \quad c=\alpha-\frac{\beta^{2}(2 p+1)}{\lambda(p+2)^{2}(p+1)},
\end{gathered}
$$

where the exponent $p \neq-2,-1$, and $\epsilon= \pm 1$.

3.1. The Jacobi Elliptic Function Solutions to (1) in the Case of $p=1$. In order to make that wave number $\mu$ a realvalued number, we have to choose constants $\lambda$ and modulus $r$ ( $c_{2}$ depends on $r$ ) to satisfy some restrictions. However, in this section, we allow $\mu$ to take values in complex number domain. From the expression of $g_{1}, g_{2}, \mu$, and $c$ in (13) and the solutions listed in Table 1, we derive the following Jacobi elliptic function solutions for (1) in the case $p=1$ :

$$
\begin{aligned}
u_{1.1}(x, t)= & -\frac{\beta}{2 \lambda}+\frac{\epsilon \beta r}{2 \lambda} \sqrt{\frac{2}{r^{2}+1}} \\
& \times \operatorname{sn}\left[\frac{\beta}{2} \sqrt{-\frac{1}{3 \lambda\left(r^{2}+1\right)}}\left(x-\alpha t+\frac{\beta^{2}}{6 \lambda} t\right)\right], \\
u_{1.2}(x, t)= & \left.-\frac{\beta}{2 \lambda}+\frac{\epsilon \beta r}{2 \lambda} \sqrt{\frac{2}{r^{2}+1}}\left(x-\alpha t+\frac{\beta^{2}}{6 \lambda} t\right)\right], \\
& \times \operatorname{cd}\left[\frac{\beta}{2} \sqrt{-\frac{1}{3 \lambda\left(r^{2}+1\right)}}\left(x-\frac{\beta}{2 \lambda}+\frac{\epsilon \beta r}{2 \lambda} \sqrt{\frac{2}{2 r^{2}-1}}\left(x-\alpha t+\frac{\beta^{2}}{6 \lambda} t\right)\right],\right. \\
u_{1.3}(x, t)=- & \times \operatorname{cn}\left[\frac{\beta}{2} \sqrt{\frac{1}{3 \lambda\left(2 r^{2}-1\right)}}\left(x-\alpha t+\frac{\beta^{2}}{6 \lambda} t\right)\right], \\
& \times \frac{\beta}{2 \lambda}+\frac{\epsilon \beta}{2 \lambda} \sqrt{\frac{2}{2-r^{2}}}\left[\frac{\beta}{2} \sqrt{\frac{1}{3 \lambda\left(2-r^{2}\right)}}(x, t)=\right. \\
u_{1.4}(x, t)= &
\end{aligned}
$$

$$
\begin{aligned}
u_{1.5}(x, t)= & \frac{\beta}{2 \lambda}+\frac{\epsilon \beta}{2 \lambda} \sqrt{\frac{2\left(r^{2}-1\right)}{2 r^{2}-1}} \\
& \times \operatorname{nc}\left[\frac{\beta}{2} \sqrt{\frac{1}{3 \lambda\left(2 r^{2}-1\right)}}\left(x-\alpha t+\frac{\beta^{2}}{6 \lambda} t\right)\right], \\
u_{1.6}(x, t)= & \left.-\frac{\beta}{2 \lambda}+\frac{\epsilon \beta}{2 \lambda} \sqrt{\frac{2}{r^{2}+1}}\left(x-\alpha t+\frac{\beta^{2}}{6 \lambda} t\right)\right], \\
& \times \operatorname{ns}\left[\frac{\beta}{2} \sqrt{-\frac{1}{3 \lambda\left(r^{2}+1\right)}}(x-\right.
\end{aligned}
$$$$
u_{1.7}(x, t)=-\frac{\beta}{2 \lambda}+\frac{\epsilon \beta}{2 \lambda} \sqrt{\frac{2}{r^{2}+1}}
$$$$
\times \mathrm{dc}\left[\frac{\beta}{2} \sqrt{-\frac{1}{3 \lambda\left(r^{2}+1\right)}}\left(x-\alpha t+\frac{\beta^{2}}{6 \lambda} t\right)\right] \text {, }
$$$$
u_{1.8}(x, t)=-\frac{\beta}{2 \lambda}+\frac{\epsilon \beta}{2 \lambda} \sqrt{\frac{2\left(r^{2}-1\right)}{r^{2}-2}}
$$$$
\times \text { nd }\left[\frac{\beta}{2} \sqrt{\frac{1}{3 \lambda\left(2-r^{2}\right)}}\left(x-\alpha t+\frac{\beta^{2}}{6 \lambda} t\right)\right] \text {, }
$$$$
u_{1.9}(x, t)=-\frac{\beta}{2 \lambda}+\frac{\epsilon \beta}{2 \lambda} \sqrt{\frac{2}{r^{2}-2}}
$$$$
\times \operatorname{cs}\left[\frac{\beta}{2} \sqrt{\frac{1}{3 \lambda\left(2-r^{2}\right)}}\left(x-\alpha t+\frac{\beta^{2}}{6 \lambda} t\right)\right] \text {, }
$$$$
u_{1.10}(x, t)=-\frac{\beta}{2 \lambda}+\frac{\epsilon \beta}{2 \lambda} \sqrt{\frac{2\left(1-r^{2}\right)}{r^{2}-2}}
$$$$
\times \operatorname{sc}\left[\frac{\beta}{2} \sqrt{\frac{1}{3 \lambda\left(2-r^{2}\right)}}\left(x-\alpha t+\frac{\beta^{2}}{6 \lambda} t\right)\right] \text {, }
$$$$
u_{1.11}(x, t)=-\frac{\beta}{2 \lambda}+\frac{\epsilon \beta r}{2 \lambda} \sqrt{\frac{2\left(r^{2}-1\right)}{1-2 r^{2}}}
$$$$
\times \operatorname{sd}\left[\frac{\beta}{2} \sqrt{-\frac{1}{3 \lambda\left(1-2 r^{2}\right)}}\left(x-\alpha t+\frac{\beta^{2}}{6 \lambda} t\right)\right] \text {, }
$$$$
u_{1.12}(x, t)=-\frac{\beta}{2 \lambda}+\frac{\epsilon \beta}{2 \lambda} \sqrt{\frac{2}{1-2 r^{2}}}
$$$$
\times \mathrm{ds}\left[\frac{\beta}{2} \sqrt{-\frac{1}{3 \lambda\left(1-2 r^{2}\right)}}\left(x-\alpha t+\frac{\beta^{2}}{6 \lambda} t\right)\right] \text {, }
$$$$
u_{1.13}(x, t)=-\frac{\beta}{2 \lambda}+\frac{\epsilon \beta}{2 \lambda} \sqrt{\frac{1}{1+r^{2}}}
$$$$
\times\left\{r \operatorname { c n } \left[\frac{\beta}{2} \sqrt{\frac{2}{3 \lambda\left(r^{2}+1\right)}}\right.\right.
$$$$
\left.\times\left(x-\alpha t+\frac{\beta^{2} t}{6 \lambda}\right)\right]
$$ 


$$
\begin{aligned}
\pm \mathrm{nd}[ & \frac{\beta}{2} \sqrt{\frac{2}{3 \lambda\left(r^{2}+1\right)}} \\
& \left.\left.\times\left(x-\alpha t+\frac{\beta^{2} t}{6 \lambda}\right)\right]\right\},
\end{aligned}
$$

$u_{1.14}(x, t)$

$$
\begin{gathered}
=-\frac{\beta}{2 \lambda}+\frac{\epsilon \beta}{2 \lambda} \sqrt{\frac{1}{2 r^{2}-1}} \\
\times\left\{1 \times\left(\operatorname { s n } \left[\frac{\beta}{2} \sqrt{\frac{2}{3 \lambda\left(1-2 r^{2}\right)}}\right.\right.\right. \\
\left.\left.\times\left(x-\alpha t+\frac{\beta^{2} t}{6 \lambda}\right)\right]\right)^{-1} \\
\pm\left(\operatorname { c n } \left[\frac{\beta}{2} \sqrt{\frac{2}{3 \lambda\left(1-2 r^{2}\right)}}\right.\right. \\
\times\left(\operatorname { s n } \left[\frac{\beta}{2} \sqrt{\frac{2}{3 \lambda\left(1-2 r^{2}\right)}}\right.\right. \\
\left.\left.\left.\times\left(x-\alpha t+\frac{\beta^{2} t}{6 \lambda}\right)\right]\right)^{-1}\right\},
\end{gathered}
$$

$u_{1.15}(x, t)$

$$
\begin{aligned}
& =-\frac{\beta}{2 \lambda}+\frac{\epsilon \beta}{2 \lambda} \sqrt{\frac{r^{2}-1}{1+r^{2}}} \\
& \times\left\{1 \times\left(\mathrm { cn } \left[\frac{\beta}{2} \sqrt{\frac{2}{3 \lambda\left(r^{2}+1\right)}}\right.\right.\right. \\
& \left.\left.\times\left(x-\alpha t+\frac{\beta^{2} t}{6 \lambda}\right)\right]\right)^{-1} \\
& \pm\left(\operatorname { s n } \left[\frac{\beta}{2} \sqrt{\frac{2}{3 \lambda\left(r^{2}+1\right)}}\right.\right. \\
& \left.\left.\times\left(x-\alpha t+\frac{\beta^{2} t}{6 \lambda}\right)\right]\right) \\
& \times\left(\operatorname { c n } \left[\frac{\beta}{2} \sqrt{\frac{2}{3 \lambda\left(r^{2}+1\right)}}\right.\right. \\
& \left.\left.\left.\times\left(x-\alpha t+\frac{\beta^{2} t}{6 \lambda}\right)\right]\right)^{-1}\right\},
\end{aligned}
$$

$$
\begin{aligned}
& u_{1.16}(x, t) \\
& =-\frac{\beta}{2 \lambda}+\frac{\epsilon \beta}{2 \lambda} \sqrt{\frac{1}{2-r^{2}}} \\
& \times\left\{1 \times\left(\operatorname { s n } \left[\frac{\beta}{2} \sqrt{\frac{2}{3 \lambda\left(r^{2}-2\right)}}\right.\right.\right. \\
& \times\left(\operatorname { d n } \left[\frac{\beta}{2} \sqrt{\frac{2}{3 \lambda\left(r^{2}-2\right)}}\right.\right. \\
& \left.\left.\times\left(x t+\frac{\beta^{2} t}{6 \lambda}\right)\right]\right)^{-1} \\
& \left.\times\left(\operatorname{sn}\left[\frac{\beta}{2} \sqrt{\frac{2}{3 \lambda\left(r^{2}-2\right)}} \frac{\beta^{2} t}{6 \lambda}\right)\right]\right)^{-1} \\
& \left.\left.\left.\times\left(x-\alpha t+\frac{\beta^{2} t}{6 \lambda}\right)\right]\right)^{-1}\right\}
\end{aligned}
$$

$u_{1.17}(x, t)$

$$
\begin{aligned}
= & -\frac{\beta}{2 \lambda}+\frac{\epsilon \beta r}{2 \lambda} \sqrt{\frac{1}{2-r^{2}}} \\
& \times\left\{\operatorname{sn}\left[\frac{\beta}{2} \sqrt{\frac{2}{3 \lambda\left(r^{2}-2\right)}}\left(x-\alpha t+\frac{\beta^{2} t}{6 \lambda}\right)\right]\right.
\end{aligned}
$$$$
\left. \pm i \mathrm{cn}\left[\frac{\beta}{2} \sqrt{\frac{2}{3 \lambda\left(r^{2}-2\right)}}\left(x-\alpha t+\frac{\beta^{2} t}{6 \lambda}\right)\right]\right\},
$$

$u_{1.18}(x, t)$

$$
=-\frac{\beta}{2 \lambda}+\frac{\epsilon \beta r}{2 \lambda} \sqrt{\frac{1}{2-r^{2}}}
$$$$
\times\left(\left(\operatorname{dn}\left[\frac{\beta}{2} \sqrt{\frac{2}{3 \lambda\left(r^{2}-2\right)}}\left(x-\alpha t+\frac{\beta^{2} t}{6 \lambda}\right)\right]\right)\right.
$$$$
\times\left(\sqrt { 1 - r ^ { 2 } } \operatorname { s n } \left[\frac{\beta}{2} \sqrt{\frac{2}{3 \lambda\left(r^{2}-2\right)}}\right.\right.
$$

$$
\left.\times\left(x-\alpha t+\frac{\beta^{2} t}{6 \lambda}\right)\right]
$$$$
\pm \mathrm{cn}\left[\frac{\beta}{2} \sqrt{\frac{2}{3 \lambda\left(r^{2}-2\right)}}\right.
$$

$$
\left.\left.\left.\times\left(x-\alpha t+\frac{\beta^{2} t}{6 \lambda}\right)\right]\right)^{-1}\right),
$$




$$
\begin{aligned}
& u_{1.19}(x, t) \\
& =-\frac{\beta}{2 \lambda}+\frac{\epsilon \beta}{2 \lambda} \sqrt{\frac{1}{2 r^{2}-1}} \\
& \quad \times\left\{r \operatorname{sn}\left[\frac{\beta}{2} \sqrt{\frac{2}{3 \lambda\left(1-2 r^{2}\right)}}\left(x-\alpha t+\frac{\beta^{2} t}{6 \lambda}\right)\right]\right. \\
& \left. \pm i \operatorname{dn}\left[\frac{\beta}{2} \sqrt{\frac{2}{3 \lambda\left(1-2 r^{2}\right)}}\left(x-\alpha t+\frac{\beta^{2} t}{6 \lambda}\right)\right]\right\},
\end{aligned}
$$

$u_{1.20}(x, t)$

$$
\begin{gathered}
=-\frac{\beta}{2 \lambda}+\frac{\epsilon \beta}{2 \lambda} \sqrt{\frac{1}{2 r^{2}-1}} \\
\times\left(\left(\operatorname{sn}\left[\frac{\beta}{2} \sqrt{\frac{1}{3 \lambda\left(1-2 r^{2}\right)}}\left(x-\alpha t+\frac{\beta^{2} t}{6 \lambda}\right)\right]\right)\right. \\
\times\left(1 \pm \operatorname{cn}\left[\frac{\beta}{2} \sqrt{\frac{2}{3 \lambda\left(1-2 r^{2}\right)}}\right.\right. \\
\left.\left.\left.\times\left(x-\alpha t+\frac{\beta^{2} t}{6 \lambda}\right)\right]\right)^{-1}\right),
\end{gathered}
$$

$u_{1.21}(x, t)$

$$
\begin{gathered}
=-\frac{\beta}{2 \lambda}+\frac{\epsilon \beta}{2 \lambda} \sqrt{\frac{r^{2}}{2-r^{2}}} \\
\times\left(\left(\operatorname{sn}\left[\frac{\beta}{2} \sqrt{\frac{1}{3 \lambda\left(r^{2}-2\right)}}\left(x-\alpha t+\frac{\beta^{2} t}{6 \lambda}\right)\right]\right)\right. \\
\times\left(1 \pm \operatorname{dn}\left[\frac{\beta}{2} \sqrt{\frac{2}{3 \lambda\left(r^{2}-2\right)}}\right.\right. \\
\left.\left.\left.\times\left(x-\alpha t+\frac{\beta^{2} t}{6 \lambda}\right)\right]\right)^{-1}\right),
\end{gathered}
$$

$u_{1.22}(x, t)$

$$
\begin{gathered}
=-\frac{\beta}{2 \lambda}+\frac{\epsilon \beta}{2 \lambda} \sqrt{\frac{1-r^{2}}{r^{2}+1}} \\
\times\left(\left(\operatorname { d n } \left[\frac{\beta}{2} \sqrt{\left.\left.\frac{2}{3 \lambda\left(r^{2}+1\right)}\left(x-\alpha t+\frac{\beta^{2} t}{6 \lambda}\right)\right]\right)}\right.\right.\right. \\
\times\left(1 \pm r \operatorname{sn}\left[\frac{\beta}{2} \sqrt{\frac{2}{3 \lambda\left(r^{2}+1\right)}}\right.\right. \\
\left.\left.\left.\times\left(x-\alpha t+\frac{\beta^{2} t}{6 \lambda}\right)\right]\right)^{-1}\right),
\end{gathered}
$$

$$
\begin{aligned}
& u_{1.23}(x, t) \\
& =-\frac{\beta}{2 \lambda}+\frac{\epsilon \beta}{2 \lambda} \sqrt{\frac{r^{2}-1}{1+r^{2}}} \\
& \times\left(\left(\operatorname{cn}\left[\frac{\beta}{2} \sqrt{\frac{2}{3 \lambda\left(r^{2}+1\right)}}\left(x-\alpha t+\frac{\beta^{2} t}{6 \lambda}\right)\right]\right)\right. \\
& \times\left(1 \pm \operatorname{sn}\left[\frac{\beta}{2} \sqrt{\frac{2}{3 \lambda\left(r^{2}+1\right)}}\right.\right. \\
& \left.\left.\left.\times\left(x-\alpha t+\frac{\beta^{2} t}{6 \lambda}\right)\right]\right)^{-1}\right) \\
& u_{1.24}(x, t) \\
& =-\frac{\beta}{2 \lambda}+\frac{\epsilon \beta\left(1-r^{2}\right)}{2 \lambda} \sqrt{-\frac{1}{r^{2}+1}} \\
& \times\left(\left(\operatorname{sn}\left[\frac{\beta}{2} \sqrt{\frac{2}{3 \lambda\left(r^{2}+1\right)}}\left(x-\alpha t+\frac{\beta^{2} t}{6 \lambda}\right)\right]\right)\right. \\
& \times\left(\operatorname { d n } \left[\frac{\beta}{2} \sqrt{\frac{2}{3 \lambda\left(r^{2}+1\right)}}\right.\right. \\
& \left.\times\left(x-\alpha t+\frac{\beta^{2} t}{6 \lambda}\right)\right] \\
& \pm \mathrm{cn}\left[\frac{\beta}{2} \sqrt{\frac{2}{3 \lambda\left(r^{2}+1\right)}}\right. \\
& \left.\left.\left.\times\left(x-\alpha t+\frac{\beta^{2} t}{6 \lambda}\right)\right]\right)^{-1}\right), \\
& u_{1.25}(x, t) \\
& =\frac{-\beta}{2 \lambda}+\frac{\epsilon \beta r^{2}}{2 \lambda \sqrt{2-r^{2}}} \\
& \times\left(\left(\operatorname{cn}\left[\frac{\beta}{2} \sqrt{\frac{2}{3 \lambda\left(r^{2}-2\right)}}\left(x-\alpha t+\frac{\beta^{2} t}{6 \lambda}\right)\right]\right)\right. \\
& \times\left(\sqrt{1-r^{2}} \pm \operatorname{dn}\left[\frac{\beta}{2} \sqrt{\frac{2}{3 \lambda\left(r^{2}-2\right)}}\right.\right. \\
& \left.\left.\left.\times\left(x-\alpha t+\frac{\beta^{2} t}{6 \lambda}\right)\right]\right)^{-1}\right) .
\end{aligned}
$$

Remark 1. From the properties of Jacobi elliptic functions, we know that when the module $r \rightarrow 0$ or $r \rightarrow 1$, sn, cn, and 
dn degenerate into triangular or hyperbolic functions, from which we can obtain soliton and triangular function solutions of (1) in the case $p=1$. In other word, when $p=1$, we can deduce Jacobi elliptic functions solutions, soliton, and triangular function solutions for (1).

Remark 2. When $\alpha=0, k=(\beta / 2) \sqrt{2 / 3 \lambda\left(1-2 r^{2}\right)}, a_{0}=$ $-\beta / 2 \lambda$, and $a_{1}= \pm k \sqrt{-3 / 2 \lambda}, u_{1.14}(x, t)$ turns into

$$
\begin{aligned}
& u_{1.14}(x, t) \\
& =a_{0} \\
& +a_{1}\left\{\operatorname{nsk}\left[x-\left(\frac{1-2 r^{2}}{2} k^{2}+\lambda a_{0}^{2}+\beta a_{0}\right) t\right]\right. \\
& \left.\quad+\operatorname{cs} k\left[x-\left(\frac{1-2 r^{2}}{2} k^{2}+\lambda a_{0}^{2}+\beta a_{0}\right) t\right]\right\},
\end{aligned}
$$

which was obtained by Yomba [17].

Remark 3. Supposing $k=(\beta / 6) \sqrt{-1 / q v \lambda}, p=(2+$ $\sqrt{3} / 3) \sqrt{-6 q \nu \lambda}$ ( $q$ and $\nu$ are arbitrary constants). When $r \rightarrow 1$ in $u_{1.17}(x, t)$, we obtain

$$
\begin{aligned}
& u_{1.17 .1}(x, t) \\
& =a_{0}-\frac{\epsilon \beta}{12 \lambda q} \sqrt{-\frac{6}{q \nu \lambda}} \\
& \times\{ \pm \sqrt{-2 q \nu \lambda}+\sqrt{-6 q \nu \lambda}(\tanh \sqrt{-6 q \nu \lambda \xi}) \\
& \pm i \operatorname{sech} \sqrt{-6 q \nu \lambda \xi}\},
\end{aligned}
$$

where $a_{0}=-(\beta p / 2 \lambda) \sqrt{-6 q \nu \lambda}+\beta / 2 \lambda, \xi=k\left(x-\left(\lambda a_{0}^{2}+\right.\right.$ $\left.\beta a_{0}\right) t$ ). Solution (19) is in full agreement with that presented in [17] by Yomba.

3.2. The Jacobi Elliptic Function Solutions to (1) in the Case of $p=1 / 2$. Similarly as in Section 3.1, we allow $\mu$ to take values in complex number field. Making use of (14) and the solutions listed in Table 1, we obtain the following Jacobi elliptic function solutions for (1) in the case $p=1 / 2$ :

$$
\begin{aligned}
u_{2.1}(x, t) & \\
= & \left\{-\frac{2 \beta}{5 \lambda}+\frac{2 \epsilon \beta r}{5 \lambda} \sqrt{\frac{2}{r^{2}+1}}\right. \\
& \left.\times \operatorname{sn}\left[\frac{\beta}{5} \sqrt{\frac{-2}{3 \lambda\left(r^{2}+1\right)}}\left(x-\alpha t+\frac{16 \beta^{2}}{75 \lambda} t\right)\right]\right\}^{2}, \\
u_{2.2}(x, t) & \left.\left\{-\frac{2 \beta}{5 \lambda}+\frac{2 \epsilon \beta r}{5 \lambda \sqrt{\frac{2}{r^{2}+1}}}\left(x-\alpha t+\frac{16 \beta^{2}}{75 \lambda} t\right)\right]\right\}^{2},
\end{aligned}
$$

$$
\begin{aligned}
u_{2.3}(x, t) & \left\{\frac{-2 \beta}{5 \lambda}+\frac{2 \epsilon \beta r}{5 \lambda} \sqrt{\frac{2}{2 r^{2}-1}}\right. \\
& \left.\times \operatorname{cn}\left[\sqrt{\frac{2 \beta^{2}}{75 \lambda\left(2 r^{2}-1\right)}}\left(x-\alpha t+\frac{16 \beta^{2}}{75 \lambda} t\right)\right]\right\}^{2}, \\
u_{2.4}(x, t) & \left\{-\frac{2 \beta}{5 \lambda}+\frac{2 \epsilon \beta}{5 \lambda} \sqrt{\frac{2}{2-r^{2}}}\right. \\
& \left.\times \operatorname{dn}\left[\frac{\beta}{5} \sqrt{\frac{2}{3 \lambda\left(2-r^{2}\right)}}\left(x-\alpha t+\frac{16 \beta^{2}}{75 \lambda} t\right)\right]\right\}^{2},
\end{aligned}
$$$$
u_{2.5}(x, t)
$$$$
=\left\{\frac{-2 \beta}{5 \lambda}+\frac{2 \epsilon \beta}{5 \lambda} \sqrt{\frac{2 r^{2}-2}{2 r^{2}-1}}\right.
$$$$
\left.\times \mathrm{nc}\left[\sqrt{\frac{2 \beta^{2}}{75 \lambda\left(2 r^{2}-1\right)}}\left(x-\alpha t+\frac{16 \beta^{2}}{75 \lambda} t\right)\right]\right\}^{2},
$$$$
u_{2.6}(x, t)
$$$$
=\left\{-\frac{2 \beta}{5 \lambda}+\frac{2 \epsilon \beta}{5 \lambda} \sqrt{\frac{2}{r^{2}+1}}\right.
$$$$
\left.\times \mathrm{ns}\left[\frac{\beta}{5} \sqrt{-\frac{2}{3 \lambda\left(r^{2}+1\right)}}\left(x-\alpha t+\frac{16 \beta^{2}}{75 \lambda} t\right)\right]\right\}^{2},
$$$$
u_{2.7}(x, t)
$$$$
=\left\{-\frac{2 \beta}{5 \lambda}+\frac{2 \epsilon \beta}{5 \lambda} \sqrt{\frac{2}{r^{2}+1}}\right.
$$$$
\left.\times \mathrm{dc}\left[\frac{\beta}{5} \sqrt{-\frac{2}{3 \lambda\left(r^{2}+1\right)}}\left(x-\alpha t+\frac{16 \beta^{2}}{75 \lambda} t\right)\right]\right\}^{2},
$$$$
u_{2.8}(x, t)
$$$$
=\left\{\frac{-2 \beta}{5 \lambda}+\frac{2 \epsilon \beta}{5 \lambda} \sqrt{\frac{2-2 r^{2}}{2-r^{2}}}\right.
$$$$
\left.\times \text { nd }\left[\frac{\beta}{5} \sqrt{\frac{2}{3 \lambda\left(2-r^{2}\right)}}\left(x-\alpha t+\frac{16 \beta^{2}}{75 \lambda} t\right)\right]\right\}^{2} \text {, }
$$

$u_{2.9}(x, t)$

$$
=\left\{-\frac{2 \beta}{5 \lambda}+\frac{2 \epsilon \beta}{5 \lambda} \sqrt{-\frac{2}{2-r^{2}}}\right.
$$




$$
\left.\times \operatorname{cs}\left[\frac{\beta}{5} \sqrt{\frac{2}{3 \lambda\left(2-r^{2}\right)}}\left(x-\alpha t+\frac{16 \beta^{2}}{75 \lambda} t\right)\right]\right\}^{2},
$$

$u_{2.10}(x, t)$

$$
\begin{aligned}
= & \left\{\frac{-2 \beta}{5 \lambda}+\frac{2 \epsilon \beta}{5 \lambda} \sqrt{\frac{2 r^{2}-2}{2-r^{2}}}\right. \\
& \left.\times \operatorname{sc}\left[\frac{\beta}{5} \sqrt{\frac{2}{3 \lambda\left(2-r^{2}\right)}}\left(x-\alpha t+\frac{16 \beta^{2}}{75 \lambda} t\right)\right]\right\}^{2},
\end{aligned}
$$

$u_{2.11}(x, t)$

$=\left\{-\frac{2 \beta}{5 \lambda}+\frac{2 \epsilon \beta}{5 \lambda} \sqrt{\frac{2 r^{2}\left(1-r^{2}\right)}{2 r^{2}-1}}\right.$

$\left.\times \operatorname{sd}\left[\frac{\beta}{5} \sqrt{\frac{2}{3 \lambda\left(2 r^{2}-1\right)}}\left(x-\alpha t+\frac{16 \beta^{2}}{75 \lambda} t\right)\right]\right\}^{2}$,

$u_{2.12}(x, t)$

$$
=\left\{\frac{-2 \beta}{5 \lambda}+\frac{2 \epsilon \beta}{5 \lambda} \sqrt{\frac{2}{1-2 r^{2}}}\right.
$$$$
\left.\times \mathrm{ds}\left[\sqrt{\frac{2 \beta^{2}}{75 \lambda\left(2 r^{2}-1\right)}}\left(x-\alpha t+\frac{16 \beta^{2}}{75 \lambda} t\right)\right]\right\}^{2},
$$

$$
u_{2.13}(x, t)
$$

$$
\begin{gathered}
=\left\{-\frac{2 \beta}{5 \lambda}+\frac{2 \epsilon \beta}{5 \lambda} \sqrt{\frac{1}{r^{2}+1}}\right. \\
\times\left(r \operatorname{cn}\left[\frac{\beta}{5} \sqrt{\frac{4}{3 \lambda\left(r^{2}+1\right)}}\left(x-\alpha t+\frac{16 \beta^{2}}{75 \lambda} t\right)\right]\right. \\
\left.\left. \pm \operatorname{dn}\left[\frac{\beta}{5} \sqrt{\frac{4}{3 \lambda\left(r^{2}+1\right)}}\left(x-\alpha t+\frac{16 \beta^{2}}{75 \lambda} t\right)\right]\right)\right\}^{2},
\end{gathered}
$$

$u_{2.14}(x, t)$

$$
\begin{aligned}
& =\left\{-\frac{2 \beta}{5 \lambda}+\frac{2 \epsilon \beta}{5 \lambda} \sqrt{-\frac{1}{1-2 r^{2}}}\right. \\
& \times\left(1 \times\left(\operatorname { s n } \left[\frac{\beta}{5} \sqrt{\frac{4}{3 \lambda\left(-2 r^{2}+1\right)}}\right.\right.\right. \\
& \left.\left.\times\left(x-\alpha t+\frac{16 \beta^{2}}{75 \lambda} t\right)\right]\right)^{-1} \\
& \pm\left(\operatorname { c n } \left[\frac{\beta}{5} \sqrt{\frac{4}{3 \lambda\left(-2 r^{2}+1\right)}}\right.\right.
\end{aligned}
$$

$$
\left.\left.\times\left(x-\alpha t+\frac{16 \beta^{2}}{75 \lambda} t\right)\right]\right)
$$$$
\times\left(\operatorname { s n } \left[\frac{\beta}{5} \sqrt{\frac{4}{3 \lambda\left(-2 r^{2}+1\right)}}\right.\right.
$$$$
\left.\left.\left.\left.\times\left(x-\alpha t+\frac{16 \beta^{2}}{75 \lambda} t\right)\right]\right)^{-1}\right)\right\}^{2}
$$

$u_{2.15}(x, t)$

$$
=\left\{-\frac{2 \beta}{5 \lambda}+\frac{2 \epsilon \beta}{5 \lambda} \sqrt{-\frac{1-r^{2}}{r^{2}+1}}\right.
$$$$
\times\left(1 \times\left(\operatorname { c n } \left[\frac{\beta}{5} \sqrt{\frac{4}{3 \lambda\left(r^{2}+1\right)}}\right.\right.\right.
$$

$$
\left.\left.\times\left(x-\alpha t+\frac{16 \beta^{2}}{75 \lambda} t\right)\right]\right)^{-1}
$$

$$
\begin{gathered}
\pm\left(\operatorname { s n } \left[\frac{\beta}{5} \sqrt{\frac{4}{3 \lambda\left(r^{2}+1\right)}}\right.\right. \\
\left.\left.\times\left(x-\alpha t+\frac{16 \beta^{2}}{75 \lambda} t\right)\right]\right) \\
\times\left(\operatorname { c n } \left[\frac{\beta}{5} \sqrt{\frac{4}{3 \lambda\left(r^{2}+1\right)}}\right.\right. \\
\left.\left.\left.\left.\times\left(x-\alpha t+\frac{16 \beta^{2}}{75 \lambda} t\right)\right]\right)^{-1}\right)\right\}^{2},
\end{gathered}
$$

$$
\begin{aligned}
u_{2.16}(x, t) & \left\{-\frac{2 \beta}{5 \lambda}+\frac{2 \epsilon \beta}{5 \lambda} \sqrt{-\frac{1}{r^{2}-2}}\right. \\
\times & \left(1 \times\left(\operatorname { s n } \left[\frac{\beta}{5} \sqrt{\frac{4}{3 \lambda\left(r^{2}-2\right)}}\right.\right.\right. \\
\pm & \left.\times\left(\operatorname{dn}\left[\frac{\beta}{5} \sqrt{\frac{4}{3 \lambda\left(r^{2}-2\right)}} \frac{16 \beta^{2}}{75 \lambda} t\right)\right]\right)^{-1} \\
\times\left(\operatorname { s n } \left[\frac{\beta}{5} \sqrt{\frac{4}{3 \lambda\left(r^{2}-2\right)}}\right.\right. & \times\left(x \beta^{2}\right. \\
& \times(5 t)])
\end{aligned}
$$




$$
\left.\left.\left.\left.\times\left(x-\alpha t+\frac{16 \beta^{2}}{75 \lambda} t\right)\right]\right)^{-1}\right)\right\}^{2}
$$

$u_{2.17}(x, t)$

$$
\begin{gathered}
=\left\{-\frac{2 \beta}{5 \lambda}+\frac{2 \epsilon \beta r}{5 \lambda} \sqrt{-\frac{1}{r^{2}-2}}\right. \\
\times\left(\operatorname { s n } \left[\frac{\beta}{5} \sqrt{\frac{4}{3 \lambda\left(r^{2}-2\right)}}\right.\right. \\
\left.\times\left(x-\alpha t+\frac{16 \beta^{2}}{75 \lambda} t\right)\right] \\
\pm i \mathrm{cn}\left[\frac{\beta}{5} \sqrt{\frac{4}{3 \lambda\left(r^{2}-2\right)}}\right. \\
\left.\left.\left.\times\left(x-\alpha t+\frac{16 \beta^{2}}{75 \lambda} t\right)\right]\right)\right\}^{2},
\end{gathered}
$$

$u_{2.18}(x, t)$

$$
=\left\{-\frac{2 \beta}{5 \lambda}+\frac{2 \epsilon \beta r}{5 \lambda} \sqrt{-\frac{1}{r^{2}-2}}\right.
$$$$
\times\left(\left(\operatorname { d n } \left[\frac{\beta}{5} \sqrt{\frac{4}{3 \lambda\left(r^{2}-2\right)}}\right.\right.\right.
$$$$
\left.\left.\times\left(x-\alpha t+\frac{16 \beta^{2}}{75 \lambda} t\right)\right]\right)
$$$$
\times\left(\sqrt { 1 - r ^ { 2 } } \mathrm { sn } \left[\sqrt{\frac{4 \beta^{2}}{75 \lambda\left(r^{2}-2\right)}}\right.\right.
$$$$
\left.\times\left(x-\alpha t+\frac{16 \beta^{2}}{75 \lambda} t\right)\right]
$$$$
\pm \mathrm{cn}\left[\sqrt{\frac{4 \beta^{2}}{75 \lambda\left(r^{2}-2\right)}}\right.
$$$$
\left.\left.\left.\left.\times\left(x-\alpha t+\frac{16 \beta^{2}}{75 \lambda} t\right)\right]\right)^{-1}\right)\right\}^{2},
$$

$$
\begin{aligned}
& u_{2.19}(x, t) \\
& =\left\{-\frac{2 \beta}{5 \lambda}+\frac{2 \epsilon \beta}{5 \lambda \sqrt{2 r^{2}-1}}\right.
\end{aligned}
$$

$$
\begin{gathered}
\times\left(\left(\operatorname { s n } \left[\frac{\beta}{5} \sqrt{\frac{4}{3 \lambda\left(1-2 r^{2}\right)}}\right.\right.\right. \\
\left.\left.\times\left(x-\alpha t+\frac{16 \beta^{2}}{75 \lambda} t\right)\right]\right) \\
\times\left(1 \pm \mathrm{cn}\left[\frac{\beta}{5} \sqrt{\frac{4}{3 \lambda\left(1-2 r^{2}\right)}}\right.\right. \\
\left.\left.\left.\left.\times\left(x-\alpha t+\frac{16 \beta^{2}}{75 \lambda} t\right)\right]\right)^{-1}\right)\right\}^{2},
\end{gathered}
$$

$$
\begin{aligned}
& u_{2.20}(x, t) \\
& =\left\{-\frac{2 \beta}{5 \lambda}+\frac{2 \epsilon \beta}{5 \lambda} \sqrt{\frac{1}{2 r^{2}-1}}\right. \\
& \times\left(r \operatorname { s n } \left[\frac{\beta}{5} \sqrt{\frac{4}{3 \lambda\left(1-2 r^{2}\right)}}\right.\right. \\
& \left.\times\left(x-\alpha t+\frac{16 \beta^{2}}{75 \lambda} t\right)\right] \\
& \pm i \operatorname{dn}\left[\frac{\beta}{5} \sqrt{\frac{4}{3 \lambda\left(1-2 r^{2}\right)}} t\right) \\
& \left.\left.\left.\times\left(x-\alpha t+\frac{16 \beta^{2}}{75 \lambda} t\right)\right]\right)\right\}^{2},
\end{aligned}
$$

$u_{2.21}(x, t)$

$$
=\left\{-\frac{2 \beta}{5 \lambda}+\frac{2 \epsilon \beta r}{5 \lambda} \sqrt{\frac{1}{2-r^{2}}}\right.
$$$$
\times\left(\left(\operatorname { s n } \left[\frac{\beta}{5} \sqrt{\frac{4}{3 \lambda\left(2-r^{2}\right)}}\right.\right.\right.
$$$$
\left.\left.\times\left(x-\alpha t+\frac{16 \beta^{2}}{75 \lambda} t\right)\right]\right)
$$

$$
\times\left(1 \pm \operatorname{dn}\left[\frac{\beta}{5} \sqrt{\frac{4}{3 \lambda\left(2-r^{2}\right)}}\right.\right.
$$

$$
\left.\left.\left.\left.\times\left(x-\alpha t+\frac{16 \beta^{2}}{75 \lambda} t\right)\right]\right)^{-1}\right)\right\}^{2},
$$

$$
\begin{aligned}
u_{2.22}(x, t) \\
=\left\{-\frac{2 \beta}{5 \lambda}+\frac{2 \epsilon \beta}{5 \lambda} \sqrt{\frac{1-r^{2}}{r^{2}+1}}\right.
\end{aligned}
$$




$$
\begin{gathered}
\times\left(\left(\operatorname { d n } \left[\frac{\beta}{5} \sqrt{\frac{4}{3 \lambda\left(r^{2}+1\right)}}\right.\right.\right. \\
\left.\left.\times\left(x-\alpha t+\frac{16 \beta^{2}}{75 \lambda} t\right)\right]\right) \\
\times\left(1 \pm r \operatorname{sn}\left[\frac{\beta}{5} \sqrt{\frac{4}{3 \lambda\left(r^{2}+1\right)}}\right.\right. \\
\left.\left.\left.\left.\times\left(x-\alpha t+\frac{16 \beta^{2}}{75 \lambda} t\right)\right]\right)^{-1}\right)\right\}^{2}
\end{gathered}
$$

$u_{2.23}(x, t)$

$$
\begin{aligned}
& =\left\{-\frac{2 \beta}{5 \lambda}+\frac{2 \epsilon \beta}{5 \lambda} \sqrt{\frac{r^{2}-1}{r^{2}+1}}\right. \\
& \times\left(\left(\operatorname { c n } \left[\frac{\beta}{5} \sqrt{\frac{4}{3 \lambda\left(r^{2}+1\right)}}\right.\right.\right. \\
& \left.\left.\times\left(x-\alpha t+\frac{16 \beta^{2}}{75 \lambda} t\right)\right]\right) \\
& \times\left(1 \pm \operatorname{sn}\left[\frac{\beta}{5} \sqrt{\frac{4}{3 \lambda\left(r^{2}+1\right)}}\right.\right. \\
& \left.\left.\left.\left.\times\left(x-\alpha t+\frac{16 \beta^{2}}{75 \lambda} t\right)\right]\right)^{-1}\right)\right\}^{2}
\end{aligned}
$$

$u_{2.24}(x, t)$

$$
\begin{aligned}
& =\left\{-\frac{2 \beta}{5 \lambda}+\frac{2 \epsilon \beta\left(1-r^{2}\right)}{5 \lambda} \sqrt{-\frac{1}{r^{2}+1}}\right. \\
& \times\left(\left(\operatorname { s n } \left[\frac{\beta}{5} \sqrt{\frac{4}{3 \lambda\left(r^{2}+1\right)}}\right.\right.\right. \\
& \left.\left.\times\left(x-\alpha t+\frac{16 \beta^{2}}{75 \lambda} t\right)\right]\right) \\
& \times\left(\operatorname { d n } \left[\frac{\beta}{5} \sqrt{\frac{4}{3 \lambda\left(r^{2}+1\right)}}\right.\right. \\
& \left.\times\left(x-\alpha t+\frac{16 \beta^{2}}{75 \lambda} t\right)\right] \\
& \pm \mathrm{cn}\left[\frac{\beta}{5} \sqrt{\frac{4}{3 \lambda\left(r^{2}+1\right)}}\right. \\
& \left.\left.\left.\left.\times\left(x-\alpha t+\frac{16 \beta^{2}}{75 \lambda} t\right)\right]\right)^{-1}\right)\right\}^{2},
\end{aligned}
$$

$$
\begin{aligned}
u_{2.25}(x, t) & \left\{-\frac{2 \beta}{5 \lambda}+\frac{2 \epsilon \beta r^{2}}{5 \lambda} \sqrt{\frac{1}{2-r^{2}}}\right. \\
& \times\left(\left(\operatorname { c n } \left[\frac{\beta}{5} \sqrt{\frac{4}{3 \lambda\left(r^{2}-2\right)}}\right.\right.\right. \\
& \left.\left.\times\left(x-\alpha t+\frac{16 \beta^{2}}{75 \lambda} t\right)\right]\right) \\
& \times\left(\sqrt{1-r^{2}} \pm \operatorname{dn}\left[\frac{\beta}{5} \sqrt{\frac{4}{3 \lambda\left(r^{2}-2\right)}}\right.\right. \\
& \left.\left.\left.\left.\times\left(x-\alpha t+\frac{16 \beta^{2}}{75 \lambda} t\right)\right]\right)^{-1}\right)\right\}^{2}
\end{aligned}
$$

When $p=1 / 2$, we can also obtain Jacobi elliptic functions solutions, soliton, and triangular function solutions for (1).

3.3. The Jacobi Elliptic Function Solutions to (1) in the Case of $p=2$. From the expressions of (15), we get the following jacobi elliptic function solutions to (1):

$$
\begin{aligned}
u_{3.1}(x, t) & \left\{-\frac{5 \beta}{8 \lambda}+\frac{\epsilon \mu r}{2} \sqrt{-\frac{15}{\lambda}}\right. \\
& \left.\times \operatorname{sn}\left[\mu\left(x-\alpha t+\frac{5 \beta^{2}}{32 \lambda} t+\frac{t}{4}\left(r^{2}+1\right) \mu^{2}\right)\right]\right\}^{1 / 2} \\
u_{3.2}(x, t) & \left\{-\frac{5 \beta}{8 \lambda}+\frac{\epsilon \mu r}{2} \sqrt{-\frac{15}{\lambda}}\left(\frac{-5\left(r^{2}+1\right)+5 \epsilon\left(r^{2}-1\right)}{6 \lambda}\right)^{1 / 2}\right. \\
= & \times-\frac{5 \beta}{8 \lambda}+\frac{\epsilon \mu r}{2} \sqrt{\frac{15}{\lambda}} \\
& \left.\times \mathrm{cd}\left[\mu\left(x-\alpha t+\frac{5 \beta^{2}}{32 \lambda} t+\frac{t}{4}\left(r^{2}+1\right) \mu^{2}\right)\right]\right\}^{1 / 2} \\
u_{3.3}(x, t) & \mu= \pm \frac{\beta}{4 r}\left(\frac{-5\left(r^{2}+1\right)+5 \epsilon\left(r^{2}-1\right)}{6 \lambda}\right)^{1 / 2},
\end{aligned}
$$


Abstract and Applied Analysis

11

$$
\begin{aligned}
& \mu= \pm \frac{\beta}{4 r}\left(\frac{5\left(2 r^{2}-1\right)+5 \epsilon}{6\left(r^{2}-1\right) \lambda}\right)^{1 / 2}, \\
& \mu= \pm \frac{\beta}{4}\left(\frac{5\left(2-r^{2}\right)+5 \epsilon r^{2}}{6\left(1-r^{2}\right) \lambda}\right)^{1 / 2} \\
& u_{3.4}(x, t) \\
& u_{3.9}(x, t) \\
& =\left\{-\frac{5 \beta}{8 \lambda}+\frac{\epsilon \mu}{2} \sqrt{\frac{15}{\lambda}}\right. \\
& =\left\{-\frac{5 \beta}{8 \lambda}+\frac{\epsilon \mu}{2} \sqrt{-\frac{15}{\lambda}}\right. \\
& \left.\times \operatorname{dn}\left[\mu\left(x-\alpha t+\frac{5 \beta^{2}}{32 \lambda} t-\frac{t}{4}\left(2-r^{2}\right) \mu^{2}\right)\right]\right\}^{1 / 2}, \\
& \left.\times \operatorname{cs}\left[\mu\left(x-\alpha t+\frac{5 \beta^{2}}{32 \lambda} t-\frac{t}{4}\left(2-r^{2}\right) \mu^{2}\right)\right]\right\}^{1 / 2}, \\
& \mu= \pm \frac{\beta}{4}\left(\frac{5\left(2-r^{2}\right)+5 \epsilon r^{2}}{6\left(1-r^{2}\right) \lambda}\right)^{1 / 2} \\
& \mu= \pm \frac{\beta}{4}\left(\frac{5\left(2-r^{2}\right)+5 \epsilon r^{2}}{6 \lambda\left(1-r^{2}\right)}\right)^{1 / 2} \text {, } \\
& u_{3.5}(x, t) \\
& =\left\{-\frac{5 \beta}{8 \lambda}+\frac{\epsilon \mu}{2} \sqrt{-\frac{15\left(1-r^{2}\right)}{\lambda}}\right. \\
& u_{3.10}(x, t) \\
& =\left\{-\frac{5 \beta}{8 \lambda}+\frac{\epsilon \mu}{2} \sqrt{-\frac{15\left(1-r^{2}\right)}{\lambda}}\right. \\
& \left.\times \mathrm{nc}\left[\mu\left(x-\alpha t+\frac{5 \beta^{2}}{32 \lambda} t-\frac{t}{4}\left(2 r^{2}-1\right) \mu^{2}\right)\right]\right\}^{1 / 2}, \\
& \left.\times \operatorname{sc}\left[\mu\left(x-\alpha t+\frac{5 \beta^{2}}{32 \lambda} t-\frac{t}{4}\left(2-r^{2}\right) \mu^{2}\right)\right]\right\}^{1 / 2}, \\
& \mu= \pm \frac{\beta}{4 r}\left(\frac{5\left(2 r^{2}-1\right)+5 \epsilon}{6\left(r^{2}-1\right) \lambda}\right)^{1 / 2} \\
& \mu= \pm \frac{\beta}{4}\left(\frac{5\left(2-r^{2}\right)+5 \epsilon r^{2}}{6 \lambda\left(1-r^{2}\right)}\right)^{1 / 2} \text {, } \\
& u_{3.6}(x, t) \\
& =\left\{-\frac{5 \beta}{8 \lambda}+\frac{\epsilon \mu}{2} \sqrt{-\frac{15}{\lambda}}\right. \\
& \left.\times \mathrm{ns}\left[\mu\left(x-\alpha t+\frac{5 \beta^{2}}{32 \lambda} t+\frac{t}{4}\left(r^{2}+1\right) \mu^{2}\right)\right]\right\}^{1 / 2}, \\
& \mu= \pm \frac{\beta}{4 r}\left(\frac{-5\left(r^{2}+1\right)+5 \epsilon\left(r^{2}-1\right)}{6 \lambda}\right)^{1 / 2} \text {, } \\
& u_{3.7}(x, t) \\
& =\left\{-\frac{5 \beta}{8 \lambda}+\frac{\epsilon \mu}{2} \sqrt{-\frac{15}{\lambda}}\right. \\
& \left.\times \mathrm{dc}\left[\mu\left(x-\alpha t+\frac{5 \beta^{2}}{32 \lambda} t+\frac{t}{4}\left(r^{2}+1\right) \mu^{2}\right)\right]\right\}^{1 / 2}, \\
& \mu= \pm \frac{\beta}{4 r}\left(\frac{-5\left(r^{2}+1\right)+5 \epsilon\left(r^{2}-1\right)}{6 \lambda}\right)^{1 / 2} \text {, } \\
& u_{3.8}(x, t) \\
& =\left\{-\frac{5 \beta}{8 \lambda}+\frac{\epsilon \mu}{2} \sqrt{-\frac{15\left(r^{2}-1\right)}{\lambda}}\right. \\
& \left.\times \operatorname{nd}\left[\mu\left(x-\alpha t+\frac{5 \beta^{2}}{32 \lambda} t-\frac{t}{4}\left(2-r^{2}\right) \mu^{2}\right)\right]\right\}^{1 / 2}, \\
& u_{3.11}(x, t) \\
& =\left\{-\frac{5 \beta}{8 \lambda}+\frac{\epsilon \mu r}{2} \sqrt{\frac{15\left(1-r^{2}\right)}{\lambda}}\right. \\
& \left.\times \operatorname{sd}\left[\mu\left(x-\alpha t+\frac{5 \beta^{2}}{32 \lambda} t-\frac{t}{4}\left(2 r^{2}-1\right) \mu^{2}\right)\right]\right\}^{1 / 2}, \\
& \mu= \pm \frac{\beta}{4 r}\left(\frac{5\left(2 r^{2}-1\right)+5 \epsilon}{6\left(r^{2}-1\right) \lambda}\right)^{1 / 2} \\
& u_{3.12}(x, t) \\
& =\left\{-\frac{5 \beta}{8 \lambda}+\frac{\epsilon \mu}{2} \sqrt{-\frac{15}{\lambda}}\right. \\
& \left.\times \mathrm{ds}\left[\mu\left(x-\alpha t+\frac{5 \beta^{2}}{32 \lambda} t-\frac{t}{4}\left(2 r^{2}-1\right) \mu^{2}\right)\right]\right\}^{1 / 2}, \\
& \mu= \pm \frac{\beta}{4 r}\left(\frac{5\left(2 r^{2}-1\right)+5 \epsilon}{6\left(r^{2}-1\right) \lambda}\right)^{1 / 2} \text {, } \\
& u_{3.13}(x, t) \\
& =\left\{-\frac{5 \beta}{8 \lambda}+\frac{\epsilon \mu}{4} \sqrt{\frac{15}{\lambda}}\right.
\end{aligned}
$$


12

Abstract and Applied Analysis

$$
\begin{aligned}
& \times\left(r \mathrm{cn}\left[\mu\left(x-\alpha t+\frac{5 \beta^{2}}{32 \lambda} t-\frac{t}{8}\left(r^{2}+1\right) \mu^{2}\right)\right]\right. \\
& \times\left(\mathrm { cn } \left[\mu \left(x-\alpha t+\frac{5 \beta^{2}}{32 \lambda} t\right.\right.\right. \\
& \left.\left. \pm \mathrm{dn}\left[\mu\left(x-\alpha t+\frac{5 \beta^{2}}{32 \lambda} t-\frac{t}{8}\left(r^{2}+1\right) \mu^{2}\right)\right]\right)\right\}^{1 / 2}, \\
& \left.\left.\left.\left.\left.-\frac{t}{8}\left(r^{2}+1\right) \mu^{2}\right)\right]\right)^{-1}\right)\right\}^{1 / 2}, \\
& \mu= \pm \frac{\beta}{2\left(1-r^{2}\right)}\left(\frac{5\left(r^{2}+1\right)+10 \epsilon r}{3 \lambda}\right)^{1 / 2}, \\
& u_{3.14}(x, t) \\
& \mu= \pm \frac{\beta}{2\left(1-r^{2}\right)}\left(\frac{5\left(r^{2}+1\right)+10 \epsilon r}{3 \lambda}\right)^{1 / 2} \text {, } \\
& =\left\{-\frac{5 \beta}{8 \lambda}+\frac{\epsilon \mu}{4} \sqrt{-\frac{15}{\lambda}}\right. \\
& u_{3.16}(x, t) \\
& =\left\{-\frac{5 \beta}{8 \lambda}+\frac{\epsilon \mu}{4} \sqrt{-\frac{15}{\lambda}}\right. \\
& \times\left(1 \times\left(\operatorname { s n } \left[\mu \left(x-\alpha t+\frac{5 \beta^{2}}{32 \lambda} t\right.\right.\right.\right. \\
& \times\left(1 \times\left(\operatorname { s n } \left[\mu \left(x-\alpha t+\frac{5 \beta^{2}}{32 \lambda} t\right.\right.\right.\right. \\
& \left.\left.\left.-\frac{t}{8}\left(1-2 r^{2}\right)\right)\right]\right)^{-1} \\
& \left.\left.\left.-\frac{t}{8}\left(r^{2}-2\right) \mu^{2}\right)\right]\right)^{-1} \\
& \pm\left(\mathrm { cn } \left[\mu \left(x-\alpha t+\frac{5 \beta^{2}}{32 \lambda} t\right.\right.\right. \\
& \pm\left(\operatorname { d n } \left[\mu \left(x-\alpha t+\frac{5 \beta^{2}}{32 \lambda} t\right.\right.\right. \\
& \left.\left.\left.-\frac{t}{8}\left(1-2 r^{2}\right) \mu^{2}\right)\right]\right) \\
& \left.\left.\left.-\frac{t}{8}\left(r^{2}-2\right) \mu^{2}\right)\right]\right) \\
& \times\left(\operatorname { s n } \left[\mu \left(x-\alpha t+\frac{5 \beta^{2}}{32 \lambda} t\right.\right.\right. \\
& \times\left(\operatorname { s n } \left[\mu \left(x-\alpha t+\frac{5 \beta^{2}}{32 \lambda} t\right.\right.\right. \\
& \left.\left.\left.\left.\left.-\frac{t}{8}\left(1-2 r^{2}\right) \mu^{2}\right)\right]\right)^{-1}\right)\right\}^{1 / 2}, \\
& \left.\left.\left.\left.\left.-\frac{t}{8}\left(r^{2}-2\right) \mu^{2}\right)\right]\right)^{-1}\right)\right\}^{1 / 2}, \\
& \mu= \pm \frac{\beta}{2}\left(\frac{5\left(1-2 r^{2}\right)+10 \epsilon r \sqrt{r^{2}-1}}{3 \lambda}\right)^{1 / 2} \text {, } \\
& u_{3.15}(x, t) \\
& =\left\{-\frac{5 \beta}{8 \lambda}+\frac{\epsilon \mu}{4} \sqrt{-\frac{15\left(1-r^{2}\right)}{\lambda}}\right. \\
& \times\left(1 \times\left(\operatorname { c n } \left[\mu \left(x-\alpha t+\frac{5 \beta^{2}}{32 \lambda} t\right.\right.\right.\right. \\
& \left.\left.\left.-\frac{t}{8}\left(r^{2}+1\right) \mu^{2}\right)\right]\right)^{-1} \\
& \pm\left(\operatorname { d n } \left[\mu \left(x-\alpha t+\frac{5 \beta^{2}}{32 \lambda} t\right.\right.\right. \\
& \left.\left.\left.-\frac{t}{8}\left(r^{2}+1\right) \mu^{2}\right)\right]\right) \\
& u_{3.17}(x, t) \\
& =\left\{-\frac{5 \beta}{8 \lambda}+\frac{\epsilon \mu r}{4} \sqrt{-\frac{15}{\lambda}}\right. \\
& \times\left(\operatorname { s n } \left[\mu \left(x-\alpha t+\frac{5 \beta^{2}}{32 \lambda} t\right.\right.\right. \\
& \left.\left.-\frac{t}{8}\left(r^{2}-2\right) \mu^{2}\right)\right] \\
& \pm i \mathrm{cn}\left[\mu \left(x-\alpha t+\frac{5 \beta^{2}}{32 \lambda} t\right.\right. \\
& \left.\left.\left.\left.-\frac{t}{8}\left(r^{2}-2\right) \mu^{2}\right)\right]\right)\right\}^{1 / 2}, \\
& \mu= \pm \frac{\beta}{2 r^{2}}\left(\frac{5\left(r^{2}-2\right)+10 \epsilon \sqrt{1-r^{2}}}{3 \lambda}\right)^{1 / 2} \text {, }
\end{aligned}
$$


Abstract and Applied Analysis

13

$$
\begin{gathered}
u_{3.18}(x, t) \\
=\left\{-\frac{5 \beta}{8 \lambda}+\frac{\epsilon \mu r}{4} \sqrt{-\frac{15}{\lambda}}\right. \\
\times\left(\operatorname { s n } \left[\mu \left(x-\alpha t+\frac{5 \beta^{2}}{32 \lambda} t\right.\right.\right. \\
\quad\left(\sqrt { 1 - r ^ { 2 } } \mathrm { sn } \left[\mu \left(x-\alpha t+\frac{5 \beta^{2}}{32 \lambda} t\right.\right.\right. \\
\left.\left.\left.-\frac{t}{8}\left(r^{2}-2\right) \mu^{2}\right)\right]\right) \\
\mu= \pm \frac{\beta}{2 r^{2}}\left(\frac{5\left(r^{2}-2\right)+10 \epsilon \sqrt{1-r^{2}}}{3 \lambda}\right)^{1 / 2} \\
\pm \mathrm{cn}\left[\mu \left(x-\alpha t+\frac{5 \beta^{2}}{32 \lambda} t\right.\right. \\
\left.\left.\left.\left.\left.-\frac{t \mu^{2}\left(r^{2}-2\right)}{8}\right)\right]\right)^{-1}\right)\right\}^{1 / 2}
\end{gathered}
$$$$
u_{3.19}(x, t)
$$$$
=\left\{-\frac{5 \beta}{8 \lambda}+\frac{\epsilon \mu}{4} \sqrt{-\frac{15}{\lambda}}\right.
$$$$
\times\left(r \operatorname { s n } \left[\mu \left(x-\alpha t+\frac{5 \beta^{2}}{32 \lambda} t\right.\right.\right.
$$$$
\left.\left.-\frac{t}{8}\left(1-2 r^{2}\right) \mu^{2}\right)\right]
$$$$
\pm i \mathrm{dn}\left[\mu \left(x-\alpha t+\frac{5 \beta^{2}}{32 \lambda} t\right.\right.
$$$$
\left.\left.\left.\left.-\frac{t}{8}\left(1-2 r^{2}\right) \mu^{2}\right)\right]\right)\right\}^{1 / 2},
$$$$
\mu= \pm \frac{\beta}{2}\left(\frac{5\left(1-2 r^{2}\right)+10 \epsilon r \sqrt{r^{2}-1}}{3 \lambda}\right)^{1 / 2},
$$

$$
\begin{aligned}
u_{3.20}(x, t) & \left\{-\frac{5 \beta}{8 \lambda}+\frac{\epsilon \mu}{4} \sqrt{-\frac{15}{\lambda}}\right. \\
& \times\left(\left(\operatorname{sn}\left[\mu\left(x-\alpha t+\frac{5 \beta^{2}}{32 \lambda} t-\frac{t}{8}\left(1-2 r^{2}\right) \mu^{2}\right)\right]\right)\right.
\end{aligned}
$$

$$
\begin{array}{r}
\times\left(1 \pm \mathrm{cn}\left[\mu \left(x-\alpha t+\frac{5 \beta^{2}}{32 \lambda} t\right.\right.\right. \\
\left.\left.\left.\left.\left.\quad-\frac{t}{8}\left(1-2 r^{2}\right) \mu^{2}\right)\right]\right)^{-1}\right)\right\}^{1 / 2}, \\
\mu= \pm \frac{\beta}{2}\left(\frac{5\left(1-2 r^{2}\right)+10 \epsilon r \sqrt{r^{2}-1}}{3 \lambda}\right)^{1 / 2},
\end{array}
$$

$u_{3.21}(x, t)$

$$
\begin{gathered}
=\left\{-\frac{5 \beta}{8 \lambda}+\frac{\epsilon \mu r}{4} \sqrt{-\frac{15}{\lambda}}\right. \\
\times\left(\left(\operatorname{sn}\left[\mu\left(x-\alpha t+\frac{5 \beta^{2}}{32 \lambda} t-\frac{t}{8}\left(r^{2}-2\right) \mu^{2}\right)\right]\right)\right. \\
\times\left(1 \pm \operatorname{dn}\left[\mu \left(x-\alpha t+\frac{5 \beta^{2}}{32 \lambda} t\right.\right.\right. \\
\left.\left.\left.\left.\left.\quad-\frac{t}{8}\left(r^{2}-2\right) \mu^{2}\right)\right]\right)^{-1}\right)\right\}^{1 / 2},
\end{gathered}
$$$$
\mu= \pm \frac{\beta}{2 r}\left(\frac{5\left(r^{2}-2\right)+5 \epsilon \sqrt{r^{4}-5 r^{2}+4}}{3 \lambda}\right)^{1 / 2}
$$

$u_{3.22}(x, t)$

$$
\begin{gathered}
=\left\{\frac{-5 \beta}{8 \lambda}+\frac{\epsilon \mu}{4} \sqrt{\frac{15\left(1-r^{2}\right)}{\lambda}}\right. \\
\times\left(\left(\operatorname{dn}\left[\mu\left(x-\alpha t+\frac{5 \beta^{2}}{32 \lambda} t-\frac{t}{8}\left(r^{2}+1\right) \mu^{2}\right)\right]\right)\right. \\
\times\left(1 \pm r \operatorname{sn}\left[\mu \left(x-\alpha t+\frac{5 \beta^{2}}{32 \lambda} t\right.\right.\right. \\
\mu= \pm \frac{t}{2\left(1-r^{2}\right)}\left(\frac{5\left(r^{2}+1\right)+10 \epsilon r}{3 \lambda}\right)^{1 / 2}
\end{gathered}
$$

$$
\begin{aligned}
& u_{3.23}(x, t) \\
& =\left\{\begin{array}{l}
-\frac{5 \beta}{8 \lambda}+\frac{\epsilon \mu}{4} \sqrt{\frac{15\left(r^{2}-1\right)}{\lambda}} \\
\quad \times\left(\left(\operatorname{cn}\left[\mu\left(x-\alpha t+\frac{5 \beta^{2}}{32 \lambda} t-\frac{t}{8}\left(r^{2}+1\right) \mu^{2}\right)\right]\right)\right. \\
\quad \times\left(1 \pm \operatorname{sn}\left[\mu \left(x-\alpha t+\frac{5 \beta^{2}}{32 \lambda} t\right.\right.\right.
\end{array}\right.
\end{aligned}
$$




$$
\begin{array}{r}
\left.\left.\left.\left.\left.-\frac{t}{8}\left(r^{2}+1\right) \mu^{2}\right)\right]\right)^{-1}\right)\right\}^{1 / 2}, \\
\mu= \pm \frac{\beta}{2\left(1-r^{2}\right)}\left(\frac{5\left(r^{2}+1\right)+10 \epsilon r}{3 \lambda}\right)^{1 / 2},
\end{array}
$$

$u_{3.24}(x, t)$

$=\left\{-\frac{5 \beta}{8 \lambda}+\frac{\epsilon \mu\left(r^{2}-1\right)}{4} \sqrt{-\frac{15}{\lambda}}\right.$

$\times\left(\left(\operatorname{sn}\left[\mu\left(x-\alpha t+\frac{5 \beta^{2}}{32 \lambda} t-\frac{t}{8}\left(r^{2}+1\right) \mu^{2}\right)\right]\right)\right.$

$\times\left(\operatorname{dn}\left[\mu\left(x-\alpha t+\frac{5 \beta^{2}}{32 \lambda} t\right.\right.\right.$

$\left.\left.-\frac{t}{8}\left(r^{2}+1\right) \mu^{2}\right)\right]$

$\pm \mathrm{cn}\left[\mu\left(x-\alpha t+\frac{5 \beta^{2}}{32 \lambda} t\right.\right.$

$\left.\left.\left.\left.\left.-\frac{t}{8}\left(r^{2}+1\right) \mu^{2}\right)\right]\right)^{-1}\right)\right\}^{1 / 2}$

$\mu= \pm \frac{\beta}{2\left(1-r^{2}\right)}\left(\frac{5\left(r^{2}+1\right)+10 \epsilon r}{3 \lambda}\right)^{1 / 2}$,

$u_{3.25}(x, t)$

$=\left\{-\frac{5 \beta}{8 \lambda}+\frac{\epsilon \mu r^{2}}{4} \sqrt{-\frac{15}{\lambda}}\right.$

$\times\left(\left(\operatorname{cn}\left[\mu\left(x-\alpha t+\frac{5 \beta^{2}}{32 \lambda} t-\frac{t \mu^{2}\left(r^{2}-2\right)}{8}\right)\right]\right)\right.$

$\times\left(\sqrt{1-r^{2}} \pm \operatorname{dn}\left[\mu\left(x-\alpha t+\frac{5 \beta^{2}}{32 \lambda} t\right.\right.\right.$

$\left.\left.\left.\left.\left.-\frac{t \mu^{2}\left(r^{2}-2\right)}{8}\right)\right]\right)^{-1}\right)\right\}^{1 / 2}$,

$\mu= \pm \frac{\beta}{2 r^{2}}\left(\frac{5\left(r^{2}-2\right)+10 \epsilon \sqrt{1-r^{2}}}{3 \lambda}\right)^{1 / 2}$.

3.4. Soliton and Triangular Function Solutions of (1). From the expression of $2 c_{1} c_{3}=c_{2}^{2}$, we know that $r \rightarrow 0$ or $r \rightarrow$ 1. According to the properties of Jacobi elliptic functions sn, $\mathrm{cn}$, and $\mathrm{dn}$, the following degenerated soliton and triangular function solutions for (1) can be obtained:

$$
\begin{aligned}
& u_{4.1}(x, t)=\left\{-\frac{\beta(2 p+1)}{2 \lambda(p+2)}+\epsilon \frac{\beta(2 p+1)}{2 \lambda(p+2)}\right. \\
& \times \tanh \left[\frac{\beta p}{p+2} \sqrt{-\frac{2 p+1}{4 \lambda(p+1)}}\right. \\
& \left.\left.\times\left(x-\alpha t+\frac{\beta^{2}(2 p+1) t}{\lambda(p+2)^{2}(p+1)}\right)\right]\right\}^{1 / p}, \\
& u_{4.2}(x, t)=\left\{-\frac{\beta(2 p+1)}{2 \lambda(p+2)}+\epsilon \frac{\sqrt{2} \beta(2 p+1)}{2 \lambda(p+2)}\right. \\
& \times \operatorname{sech}\left[\frac{\beta p}{p+2} \sqrt{\frac{2 p+1}{2 \lambda(p+1)}}\right. \\
& \left.\left.\times\left(x-\alpha t+\frac{\beta^{2}(2 p+1) t}{\lambda(p+2)^{2}(p+1)}\right)\right]\right\}^{1 / p}, \\
& u_{4.3}(x, t)=\left\{-\frac{\beta(2 p+1)}{2 \lambda(p+2)}+\epsilon \frac{\beta(2 p+1)}{2 \lambda(p+2)}\right. \\
& \times \operatorname{coth}\left[\frac{\beta p}{p+2} \sqrt{-\frac{2 p+1}{4 \lambda(p+1)}}\right. \\
& \left.\left.\times\left(x-\alpha t+\frac{\beta^{2}(2 p+1) t}{\lambda(p+2)^{2}(p+1)}\right)\right]\right\}^{1 / p}, \\
& u_{4.4}(x, t)=\left\{-\frac{\beta(2 p+1)}{2 \lambda(p+2)}+i \epsilon \frac{\sqrt{2} \beta(2 p+1)}{2 \lambda(p+2)}\right. \\
& \times \operatorname{csch}\left[\frac{\beta p}{p+2} \sqrt{\frac{2 p+1}{2 \lambda(p+1)}}\right. \\
& \left.\left.\times\left(x-\alpha t+\frac{\beta^{2}(2 p+1) t}{\lambda(p+2)^{2}(p+1)}\right)\right]\right\}^{1 / p},
\end{aligned}
$$

$$
\begin{aligned}
& u_{4.5}(x, t) \\
& =\left\{-\frac{\beta(2 p+1)}{2 \lambda(p+2)}+\epsilon \frac{\beta(2 p+1)}{2 \lambda(p+2)}\right. \\
& \times\left(\left(\operatorname { c o s h } \left(\frac{\beta p}{p+2} \sqrt{-\frac{2 p+1}{\lambda(p+1)}}\right.\right.\right. \\
& \times(x-\alpha t \\
& \left.\left.\left.+\frac{\beta^{2}(2 p+1) t}{\lambda(p+2)^{2}(p+1)}\right)\right) \pm 1\right)
\end{aligned}
$$




$$
\begin{aligned}
& \times\left(\operatorname { s i n h } \left(\frac{\beta p}{p+2} \sqrt{-\frac{2 p+1}{\lambda(p+1)}}\right.\right. \\
& \times[x-\alpha t \\
& \times(x-\alpha t \\
& \left.\left.\left.\left.\left.+\frac{\beta^{2}(2 p+1) t}{\lambda(p+2)^{2}(p+1)}\right)\right)\right)^{-1}\right)\right\}^{1 / p} \\
& u_{4.6}(x, t) \\
& =\left\{-\frac{\beta(2 p+1)}{2 \lambda(p+2)}+\epsilon \frac{\beta(2 p+1)}{2 \lambda(p+2)}\right. \\
& \times\left(\left(\operatorname { s i n h } \left(\frac{\beta p}{p+2} \sqrt{-\frac{2 p+1}{\lambda(p+1)}}\right.\right.\right. \\
& \times[x-\alpha t \\
& \left.\left.\left.+\frac{\beta^{2}(2 p+1) t}{\lambda(p+2)^{2}(p+1)}\right]\right) \pm i\right) \\
& \times\left(\operatorname { c o s h } \left(\frac{\beta p}{p+2} \sqrt{-\frac{2 p+1}{\lambda(p+1)}}\right.\right. \\
& \times[x-\alpha t \\
& \left.\left.\left.\left.\left.+\frac{\beta^{2}(2 p+1) t}{\lambda(p+2)^{2}(p+1)}\right]\right)\right)^{-1}\right)\right\}^{1 / p}, \\
& u_{4.7}(x, t) \\
& =\left\{-\frac{\beta(2 p+1)}{2 \lambda(p+2)}+\epsilon \frac{\beta(2 p+1)}{2 \lambda(p+2)}\right. \\
& \times\left(\left(\operatorname { s i n h } \left(\frac{\beta p}{p+2} \sqrt{-\frac{2 p+1}{\lambda(p+1)}}\right.\right.\right. \\
& \times(x-\alpha t \\
& \left.\left.\left.+\frac{\beta^{2}(2 p+1) t}{\lambda(p+2)^{2}(p+1)}\right)\right)\right) \\
& u_{4.8}(x, t) \\
& =\left\{-\frac{\beta(2 p+1)}{2 \lambda(p+2)}+\epsilon \frac{\sqrt{2} \beta(2 p+1)}{2 \lambda(p+2)}\right. \\
& \left.\left.\left.\left.\left.+\frac{\beta^{2}(2 p+1) t}{\lambda(p+2)^{2}(p+1)}\right]\right)\right)^{-1}\right)\right\}^{1 / p}, \\
& \times \sec \left[\frac{\beta p}{p+2} \sqrt{-\frac{2 p+1}{2 \lambda(p+1)}}\right. \\
& \left.\left.\times\left(x-\alpha t+\frac{\beta^{2}(2 p+1) t}{\lambda(p+2)^{2}(p+1)}\right)\right]\right\}^{1 / p}, \\
& u_{4.9}(x, t) \\
& =\left\{-\frac{\beta(2 p+1)}{2 \lambda(p+2)}+\epsilon \frac{\sqrt{2} \beta(2 p+1)}{2 \lambda(p+2)}\right. \\
& \times \csc \left[\frac{\beta p}{p+2} \sqrt{-\frac{2 p+1}{2 \lambda(p+1)}}\right. \\
& \left.\left.\times\left(x-\alpha t+\frac{\beta^{2}(2 p+1) t}{\lambda(p+2)^{2}(p+1)}\right)\right]\right\}^{1 / p}, \\
& u_{4.10}(x, t) \\
& =\left\{-\frac{\beta(2 p+1)}{2 \lambda(p+2)}+i \epsilon \frac{\beta(2 p+1)}{2 \lambda(p+2)}\right. \\
& \times \cot \left[\frac{\beta p}{p+2} \sqrt{\frac{2 p+1}{4 \lambda(p+1)}}\right. \\
& \left.\left.\times\left(x-\alpha t+\frac{\beta^{2}(2 p+1) t}{\lambda(p+2)^{2}(p+1)}\right)\right]\right\}^{1 / p}, \\
& u_{4.11}(x, t) \\
& =\left\{-\frac{\beta(2 p+1)}{2 \lambda(p+2)}+i \epsilon \frac{\beta(2 p+1)}{2 \lambda(p+2)}\right. \\
& \times \tan \left[\frac{\beta p}{p+2} \sqrt{\frac{2 p+1}{4 \lambda(p+1)}}\right. \\
& \left.\left.\times\left(x-\alpha t+\frac{\beta^{2}(2 p+1) t}{\lambda(p+2)^{2}(p+1)}\right)\right]\right\}^{1 / p}, \\
& u_{4.12}(x, t) \\
& =\left\{-\frac{\beta(2 p+1)}{2 \lambda(p+2)}+i \epsilon \frac{\beta(2 p+1)}{2 \lambda(p+2)}\right.
\end{aligned}
$$




$$
\begin{gathered}
\times\left(\left(1 \pm \cos \left(\frac{\beta p}{p+2} \sqrt{\frac{2 p+1}{\lambda(p+1)}}\right.\right.\right. \\
\times(x-\alpha t \\
\left.\times\left(\sin \left(\frac{\beta p}{p+2} \sqrt{\frac{2 p+1}{\lambda(p+2)^{2}(p+1)}}\right)\right)\right) \\
\times(x-\alpha t \\
\left.\left.\left.\left.\left.+\frac{\beta^{2}(2 p+1) t}{\lambda(p+2)^{2}(p+1)}\right)\right)\right)^{-1}\right)\right\}^{1 / p},
\end{gathered}
$$$$
u_{4.13}(x, t)
$$$$
=\left\{-\frac{\beta(2 p+1)}{2 \lambda(p+2)}+i \epsilon \frac{\beta(2 p+1)}{2 \lambda(p+2)}\right.
$$$$
\times\left(\left(1 \pm \sin \left(\frac{\beta p}{p+2} \sqrt{\frac{2 p+1}{\lambda(p+1)}}\right.\right.\right.
$$$$
\times[x-\alpha t
$$$$
\left.\left.\left.+\frac{\beta^{2}(2 p+1) t}{\lambda(p+2)^{2}(p+1)}\right]\right)\right)
$$$$
\times\left(\operatorname { c o s } \left(\frac{\beta p}{p+2} \sqrt{\frac{2 p+1}{\lambda(p+1)}}\right.\right.
$$$$
\times[x-\alpha t
$$$$
\left.\left.\left.\left.\left.+\frac{\beta^{2}(2 p+1) t}{\lambda(p+2)^{2}(p+1)}\right]\right)\right)^{-1}\right)\right\}^{1 / p},
$$

$$
\begin{aligned}
u_{4.14}(x, t) & \\
= & \left\{-\frac{\beta(2 p+1)}{2 \lambda(p+2)}+i \epsilon \frac{\beta(2 p+1)}{2 \lambda(p+2)}\right. \\
& \times\left(\left(\operatorname { s i n } \left(\frac{\beta p}{p+2} \sqrt{\frac{2 p+1}{\lambda(p+1)}}\right.\right.\right.
\end{aligned}
$$

$$
\begin{aligned}
& \times(x-\alpha t \\
& \left.\left.\left.+\frac{\beta^{2}(2 p+1) t}{\lambda(p+2)^{2}(p+1)}\right)\right)\right) \\
& \times\left(1 \pm \cos \left(\frac{\beta p}{p+2} \sqrt{\frac{2 p+1}{\lambda(p+1)}}\right.\right. \\
& \times[x-\alpha t \\
& \left.\left.\left.\left.\left.+\frac{\beta^{2}(2 p+1) t}{\lambda(p+2)^{2}(p+1)}\right]\right)\right)^{-1}\right)\right\}^{1 / p}, \\
& u_{4.15}(x, t) \\
& =\left\{-\frac{\beta(2 p+1)}{2 \lambda(p+2)}+i \epsilon \frac{\beta(2 p+1)}{2 \lambda(p+2)}\right. \\
& \times\left(\left(\operatorname { c o s } \left(\frac{\beta p}{p+2} \sqrt{\frac{2 p+1}{\lambda(p+1)}}\right.\right.\right. \\
& \times[x-\alpha t \\
& \left.\left.\left.+\frac{\beta^{2}(2 p+1) t}{\lambda(p+2)^{2}(p+1)}\right]\right)\right) \\
& \times\left(1 \pm \sin \left(\frac{\beta p}{p+2} \sqrt{\frac{2 p+1}{2 \lambda(p+1)}}\right.\right. \\
& \times(x-\alpha t \\
& \left.\left.\left.\left.\left.+\frac{\beta^{2}(2 p+1) t}{\lambda(p+2)^{2}(p+1)}\right)\right)\right)^{-1}\right)\right\}^{1 / p} .
\end{aligned}
$$

Remark 4. When $r \rightarrow 0$ and $r \rightarrow 1$ in the solutions $u_{1.1}(x, t)$ to $u_{3.25}(x, t)$, the soliton and triangular function solutions we obtain can be found in $u_{4.1}(x, t)$ to $u_{4.15}(x, t)$ while $p=$ $1,2,1 / 2$, respectively. For example, when $r \rightarrow 1, u_{2.17}$ and $u_{3.17}$ can be expressed by

$$
\begin{aligned}
u_{2.17 .1}(x, t)=\{ & -\frac{2 \beta}{5 \lambda}+\frac{2 \epsilon \beta}{5 \lambda} \\
& \times\left(\tanh \left[\frac{\beta}{5} \sqrt{-\frac{4}{3 \lambda}}\left(x-\alpha t+\frac{16 \beta^{2}}{75 \lambda} t\right)\right]\right. \\
& \left.\left. \pm i \operatorname{sech}\left[\frac{\beta}{5} \sqrt{-\frac{4}{3 \lambda}}\left(x-\alpha t+\frac{16 \beta^{2}}{75 \lambda} t\right)\right]\right)\right\}^{2},
\end{aligned}
$$




$$
\begin{aligned}
u_{3.17 .1}(x, t)=\{ & -\frac{5 \beta}{8 \lambda}+\frac{5 \epsilon \beta}{8 \lambda} \\
& \times\left(\tanh \left[\frac{\beta}{2} \sqrt{-\frac{5}{3 \lambda}}\left(x-\alpha t+\frac{5 \beta^{2}}{48 \lambda} t\right)\right]\right. \\
& \left.\left. \pm i \operatorname{sech}\left[\frac{\beta}{2} \sqrt{-\frac{5}{3 \lambda}}\left(x-\alpha t+\frac{5 \beta^{2}}{48 \lambda} t\right)\right]\right)\right\}^{1 / 2},
\end{aligned}
$$

which are the same as $u_{4.6}$ in the case of $p=1 / 2,2$, respectively.

Remark 5. In these expressions of solutions, $u_{4.1}(x, t)$ and $u_{4.2}(x, t)$ are in full agreement with the result which was obtained by Zhang et al. [15].

\section{Conclusion}

In this paper, by using the auxiliary differential equation method, we obtain the Jacobi function solutions, the degenerated solitons, and the triangular function solutions to the generalized dispersive $\mathrm{KdV}-\mathrm{mKdV}$ equation (1) in the case of $p=1, p=2$, and $p=1 / 2$ with the help of symbolic computation system Matlab. However, for arbitrary exponent $p$, we only obtain the degenerated solitons and triangle function solutions to (1). It may need other technique to deal with the Jacobi elliptic function solutions for the generalized dispersive $\mathrm{KdV}$-mKdV equation with arbitrary exponent $p$.

\section{Acknowledgments}

The authors are grateful to the referees for their helpful and useful comments. This research is partially supported by the Scientific Research Fund of Chongqing Technology and Business University (no. 1355006).

\section{References}

[1] M. Wadati, "The modified Korteweg-de Vries equation," Journal of the Physical Society of Japan, vol. 34, pp. 1289-1296, 1973.

[2] M. Wadati, "Introduction to solitons," Journal of the Physical Society of Japan, vol. 57, no. 5-6, pp. 841-847, 2001.

[3] W. Malfliet and W. Hereman, "The tanh method. I. Exact solutions of nonlinear evolution and wave equations," Physica Scripta, vol. 54, no. 6, pp. 563-568, 1996.

[4] E. Fan and H. Zhang, "New exact solutions to a solutions to a system of coupled KdV equations," Physics Letters A, vol. 245, no. 5, pp. 389-392, 1998.

[5] S. Lai, Y. H. Wu, and B. Wiwatanapataphee, "On exact travelling wave solutions for two types of nonlinear $K(n, n)$ equations and a generalized KP equation," Journal of Computational and Applied Mathematics, vol. 212, no. 2, pp. 291-299, 2008.

[6] Z. Q. Liu, L. Guan, S. H. Lee, and S. M. Kang, "Existence and iterative algorithms of positive solutions for a higher order nonlinear neutral delay differential equation," Abstract and Applied Analysis, vol. 2013, Article ID 165382, 28 pages, 2013.
[7] S. Lai and Y. Wu, "The local well-posedness and existence of weak solutions for a generalized Camassa-Holm equation," Journal of Differential Equations, vol. 248, no. 8, pp. 2038-2063, 2010.

[8] J. H. He, "Periodic solution of the hematopoiesis equation," Abstract and Applied Analysis, vol. 2013, Article ID 290287, 2 pages, 2013.

[9] S. Y. Lai and B. Wiwatanapataphe, "The asymptotics of global solutions for semilinear wave equations in two space dimensions," Dynamics of Continuous, Discrete \& Impulsive Systems $B$, vol. 18, no. 5, pp. 647-657, 2011.

[10] Y. Chen, B. Li, and H. Zhang, "New exact solutions for modified nonlinear dispersive equations $m K(m, n)$ in higher dimensions spaces," Mathematics and Computers in Simulation, vol. 64, no. 5, pp. 549-559, 2004.

[11] L. Tian and X. Song, "New peaked solitary wave solutions of the generalized Camassa-Holm equation," Chaos, Solitons and Fractals, vol. 19, no. 3, pp. 621-637, 2004.

[12] Sirendaoreji and S. Jiong, "Auxiliary equation method for solving nonlinear partial differential equations," Physics Letters A, vol. 309, no. 5-6, pp. 387-396, 2003.

[13] Sirendaoreji, "Exact travelling wave solutions for four forms of nonlinear Klein-Gordon equations," Physics Letters A, vol. 363, no. 5-6, pp. 440-447, 2007.

[14] Q. Wang, Y. Chen, and H. Zhang, "An extended Jacobi elliptic function rational expansion method and its application to $(2+$ 1)-dimensional dispersive long wave equation," Physics Letters A, vol. 340, no. 5-6, pp. 411-426, 2005.

[15] S. Zhang, W. Wang, and J. L. Tong, "The improved sub-ODE method for a generalized $\mathrm{KdV}$-mKdV equation with nonlinear terms of any order," Physics Letters A, vol. 372, no. 21, pp. 38083813, 2008.

[16] H.-M. Li, "New exact solutions of nonlinear Gross-Pitaevskii equation with weak bias magnetic and time-dependent laser fields," Chinese Physics, vol. 14, no. 2, pp. 251-256, 2005.

[17] E. Yomba, "The extended Fan's sub-equation method and its application to KdV-MKdV, BKK and variant Boussinesq equations," Physics Letters A, vol. 336, no. 6, pp. 463-476, 2005. 


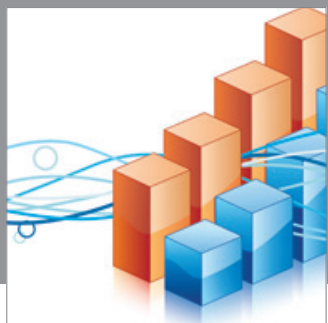

Advances in

Operations Research

mansans

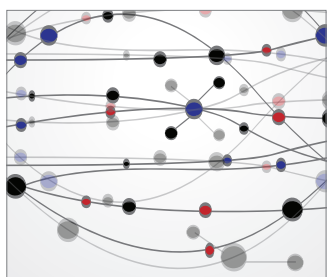

The Scientific World Journal
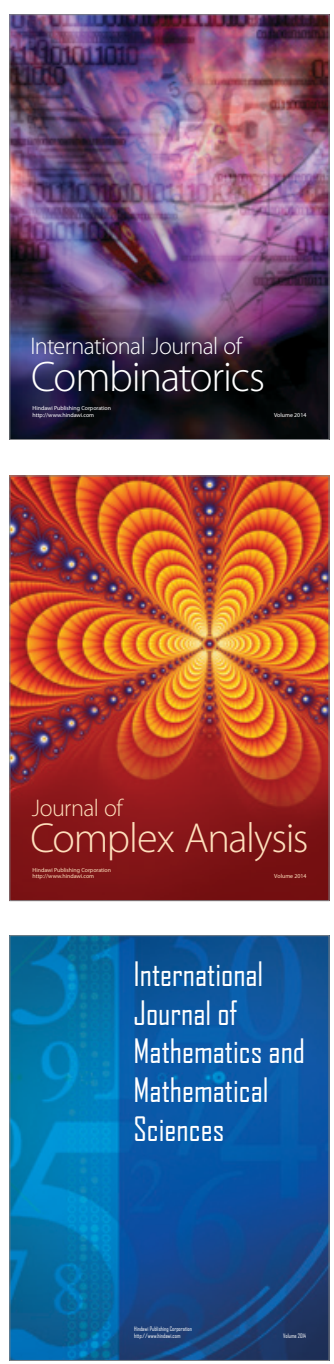
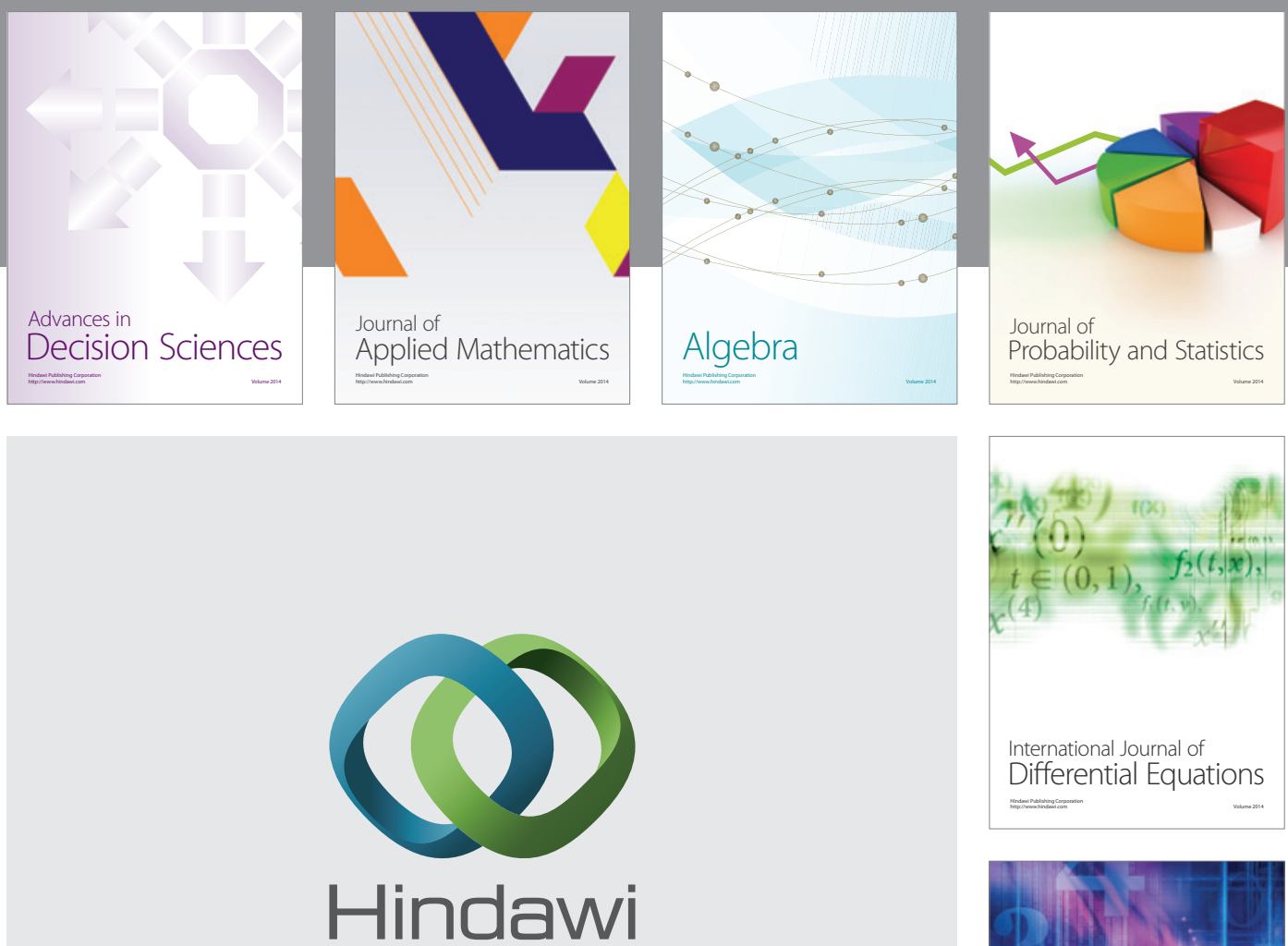

Submit your manuscripts at http://www.hindawi.com
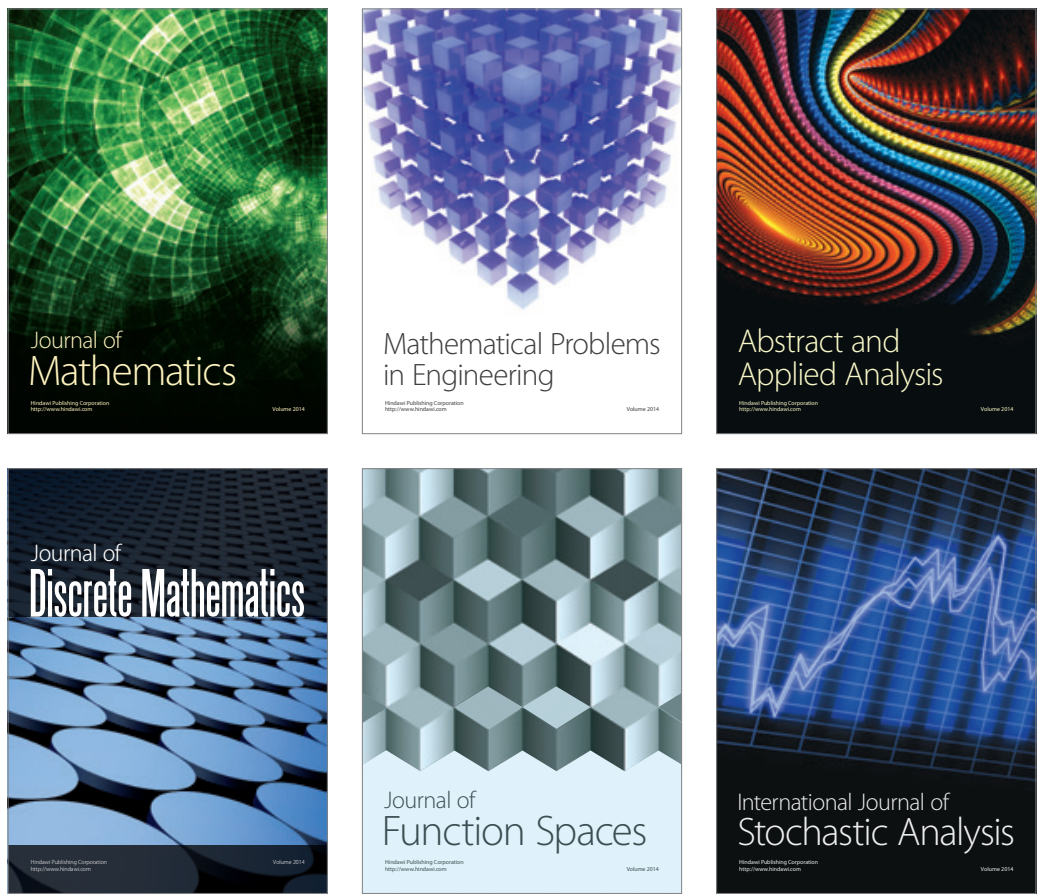

Journal of

Function Spaces

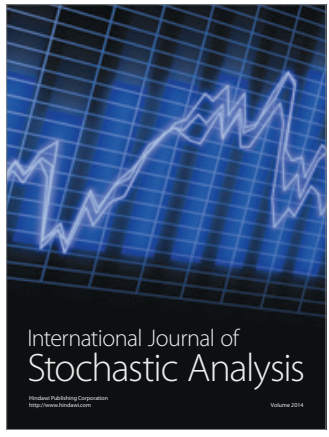

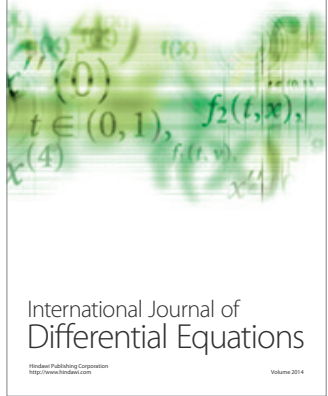
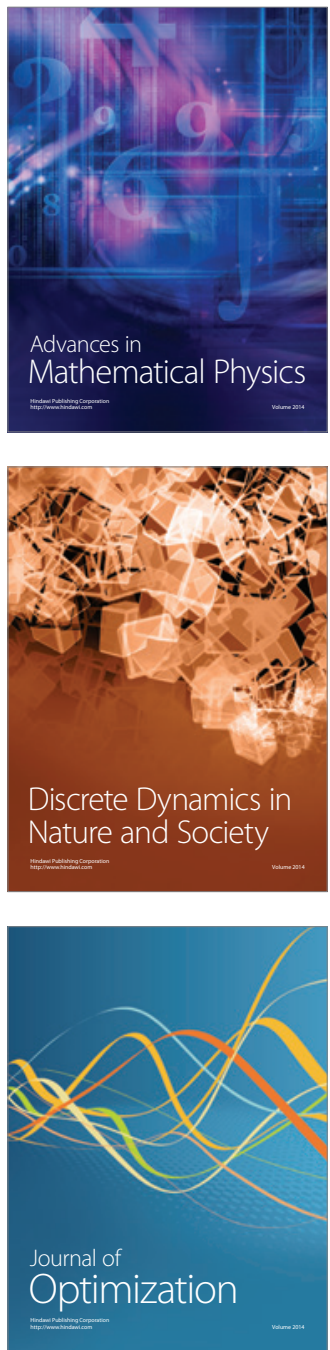